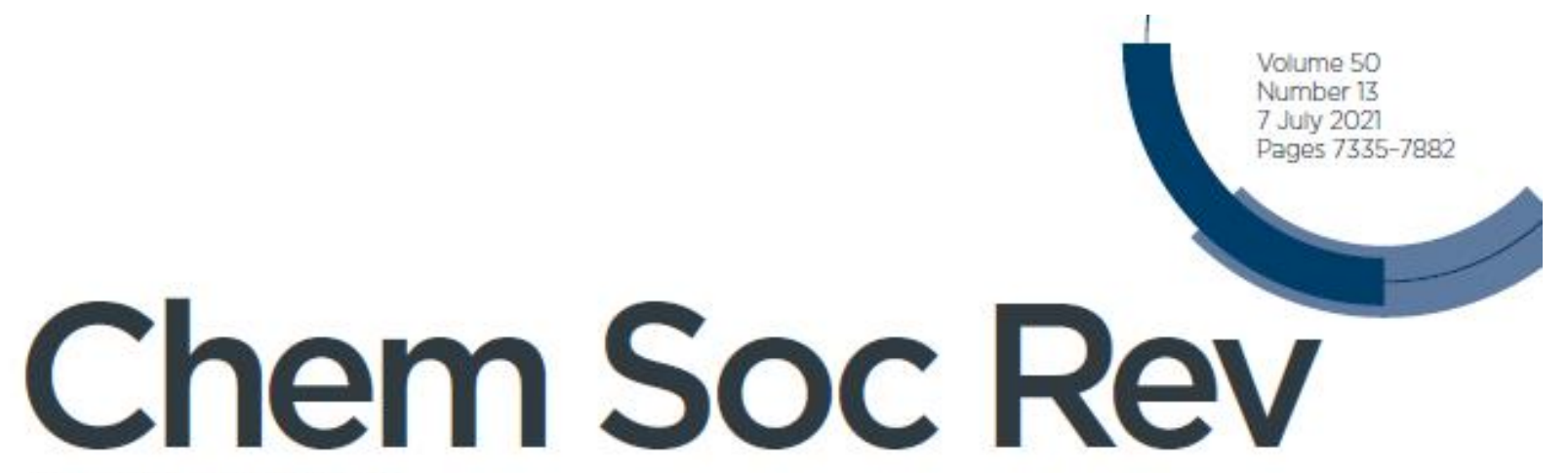

Chemical Society Reviews

rsc.li/chem-soc-rev

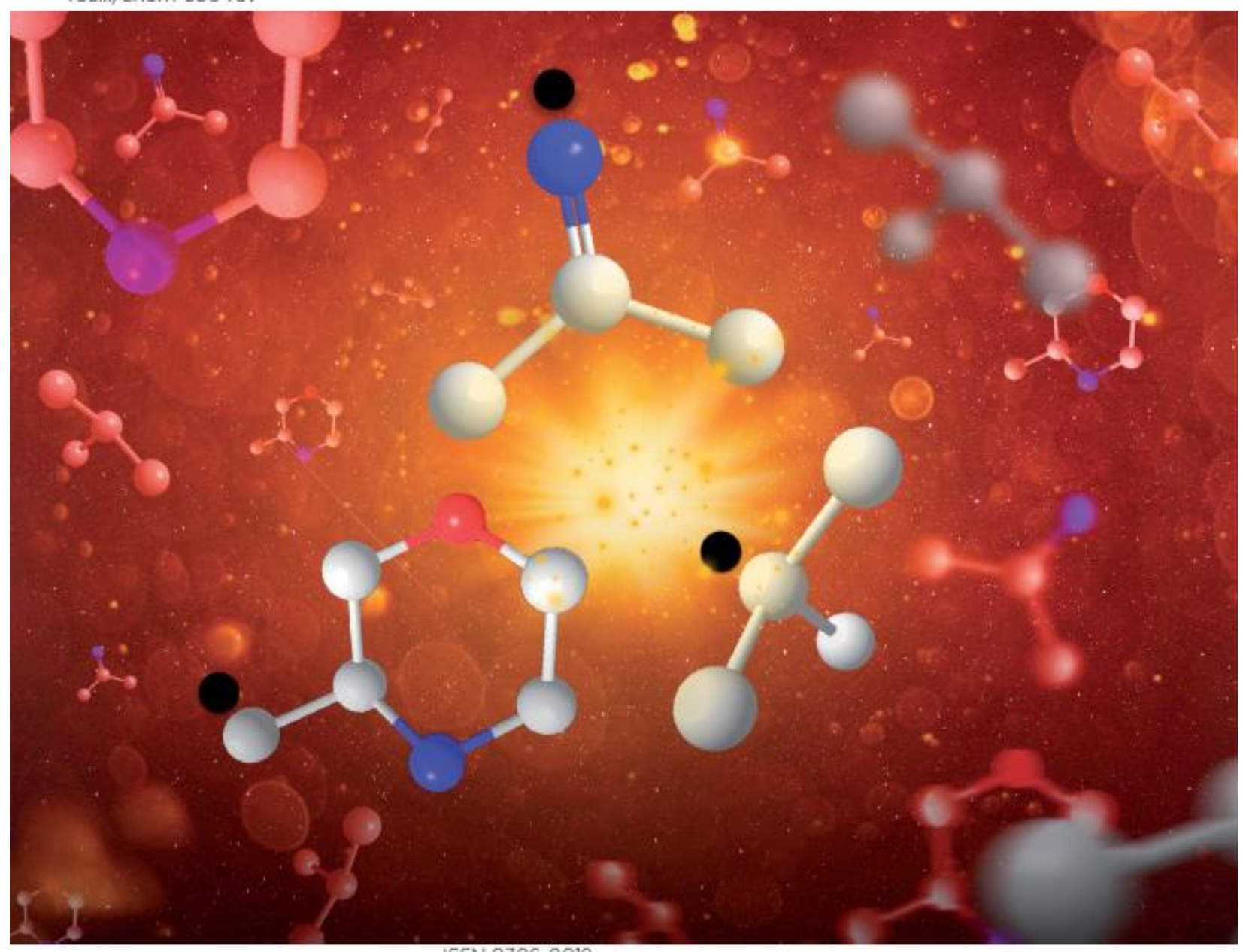

ISSN 0306-0012

ROYAL SOCIETY OF CHEMISTRY

\section{REVIEW ARTICLE}

Mohammed Latrache and Norbert Hoffmann

Photochemical radical cyclization reactions with imines,

hydrazones, oximes and related compounds 


\title{
Photochemical Radical Cyclization Reactions with Imines, Hydrazones, Oximes and Related Compounds
}

Mohammed Latrache and Norbert Hoffmann*

CNRS, Université de Reims Champagne-Ardenne, ICMR, Equipe de Photochimie, UFR Sciences, B.P. 1039, 51687 Reims, France, Phone : + 333269133 10, e-mail: norbert.hoffmann@univ-reims.fr

\begin{abstract}
Photochemical reactions are a key method to generate radical intermediates. Often under these conditions no toxic reagents are necessary. During recent years, photo-redox catalytic reactions considerably push this research domain. These reaction conditions are particularly mild and safe which enables the transformation of poly-functional substrates into complex products. The synthesis of heterocyclic compounds is particularly important since they play an important role in the research of biologically active products. In this review, photochemical radical cyclization reactions of imines and related compounds such as oximes, hydrazones and chloroimines are presented. Reaction mechanisms are discussed and the structural diversity and complexity of the products are presented. Radical intermediates are mainly generated in two ways: (1) Electronic excitation is achieved by light absorption of the substrates. (2) The application of photoredox catalysis is now systematical studied for these reactions. Recently, also excitation of charge transfer complexes have been studied in this context with many perspectives.
\end{abstract}

\section{Introduction}

Radical reactions are an interesting tool of synthetic organic chemistry and offer numerous alternatives to polar reactions. Thus, they contribute to extend the molecular diversity as it is highly appreciated, for instance, in the domain of pharmaceutical or agro chemistry. Due to the increased reactivity of intermediates, stable chemical bonds are formed in a convenient way. ${ }^{1}$ Although, polar effects play an important role in radical chemistry ${ }^{2}$, these reactions are carried out with a large variety of substrates. In the past, toxic reagents or activating groups have often been used to perform radical reactions in a selective way. ${ }^{3}$ For example, many of these transformations are carried out with tin reagents or selenium substituents on the starting molecules. Continuous efforts have been made to avoid the utilization of such reagents and to perform these reactions in a more sustainable way. ${ }^{4}$ The application of photochemical reaction conditions is a general method to generate radical species. In this context the photon is considered as a clean and traceless reagent. ${ }^{5}$ Since more than 10 years, photochemical reactions are again widely applied to organic synthesis. ${ }^{6}$ To a large extent, the recent renaissance of this research domain is based on works on photo-redox catalytic reactions and their application to organic chemistry. ${ }^{7}$ Meanwhile, a large variety of photoredox catalysts with different redox potentials for almost each functional group are described.

A lot of intramolecular radical reactions, especially cyclizations have been studied. ${ }^{8}$ They are of particular interest since a high degree of molecular complexity is generated in this way. The synthesis of 
heterocycles is an important topic in domains like pharmaceutical chemistry or material science. The photochemically induced radical cyclizations and cyccloadditions play an important role. To a large extent, they contribute to heterocyclic chemistry. ${ }^{9,10}$

Imines possess a $\mathrm{C}=\mathrm{N}$ double bond which may be easily functionalized or incorporated in heterocyclic compounds via cyclization reactions. ${ }^{11,12}$ Imines may be in chemical or more precisely in tautomeric equilibrium with enamines when mobile hydrogen atoms are located in the $\alpha$ position. ${ }^{13}$ Substitution of the nitrogen atom by a second nitrogen (hydrazones) or by an oxygen (oxime derivatives) reduces or inhibits tautomerization. Photochemical reactions of these derivatives have also been reported. ${ }^{14}$ In this regard, they may be compared to corresponding carbonyl compounds. ${ }^{15}$ One of the privileged photochemical processes is the fragmentation of the $\mathrm{N}-\mathrm{N}$ or the $\mathrm{N}-\mathrm{O}$ bond. Thus a variety of $\mathrm{N}$-centered radicals are generated.

\section{Imines}

\subsection{Generation of radical intermediates by electronic excitation of the substrates}

Radical cyclisation reactions with electronically excited imines have been much less studied when compared to their carbonyl analogues. UV irradiation of imine functions provides an efficient way to access $\mathrm{N}$-centered radical species which can initiate radical cyclization chemistry. ${ }^{16,17}$

Recently, Stephenson et al. reported a masked N-centered radical strategy designed to supply 1aminonorbornanes $\mathbf{3}$ from the Schiff bases of aminocyclopropanes $\mathbf{1}$ by initiating a multi-step radical fragmentation/ cyclization sequence forming two new C-C bonds (Scheme 1). ${ }^{18}$ The treatment of cyclopropylimine such as 1 with violet light $(390 \mathrm{~nm})$ results in the formation of the $S_{1}\left(n, \pi^{*}\right)$ excited state diyl 4. The strain driven ring opening of the cyclopropane is induced by the N-centered radical. In the resulting diradical intermediate 5, a 6 -exo-trig cyclization at the alkene moiety followed by a 5-exo-

trig cyclization radical combination process (6) occur leading to the final product 2 . It should be pointed out that the imine function is regenerated in this step. In this synthesis, the imine is both introduced (1) and removed (3) via simple and mild transformations. 


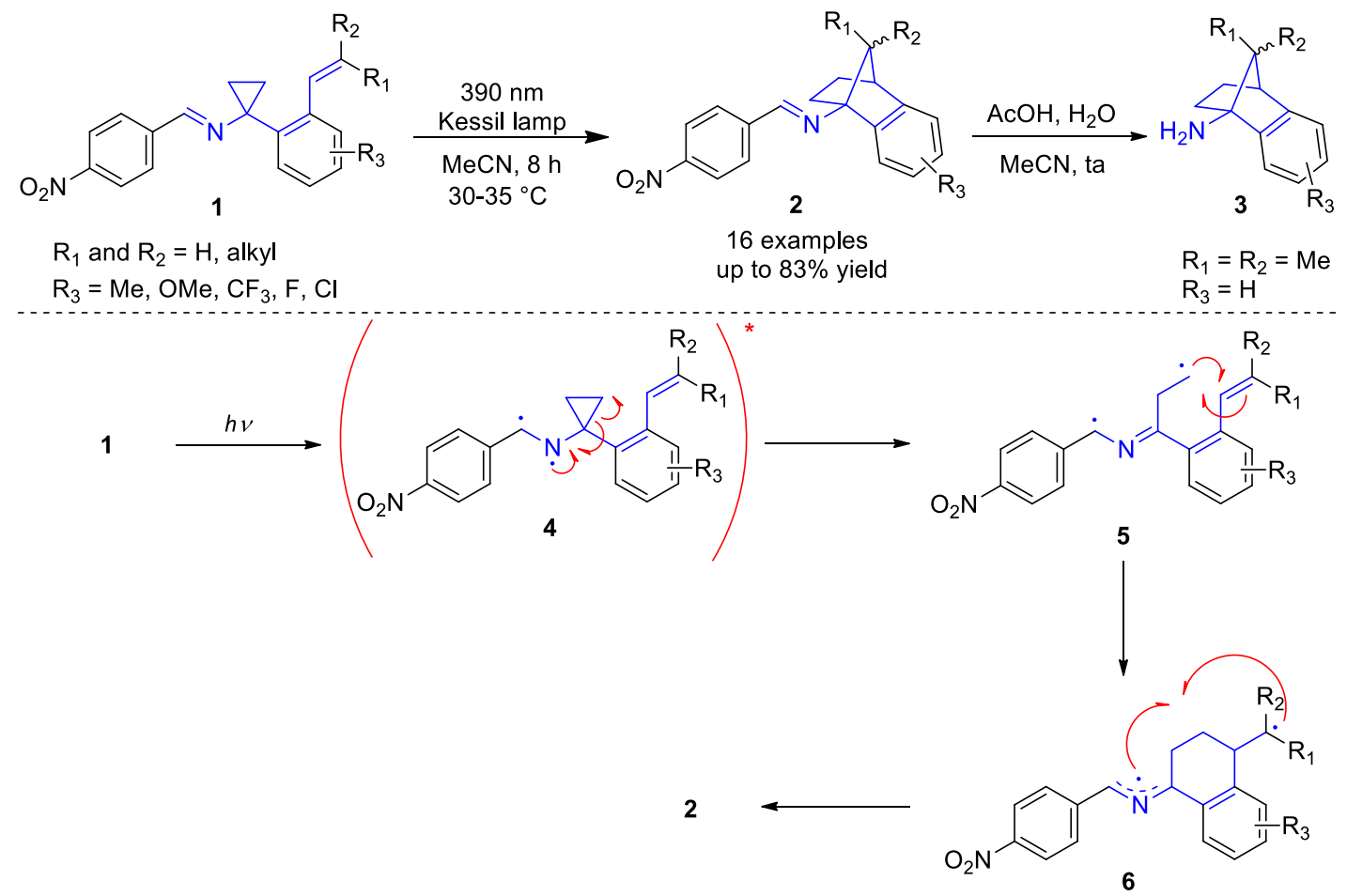

Scheme 1: Photochemically generated masked N-centered radicals for the synthesis of 1aminonorbornanes and the proposed mechanism.

A variety of cyclopropylimines with fused aryl and heteroaryl rings has been used to access to the corresponding Schiff base-protected 1-aminonorbornanes in good yields. In this reaction, the photochemical rearrangement of $\mathrm{N}$-cyclopropylimines to 1 -pyrrolines could be achieved. ${ }^{19,20}$ However, this reaction was not observed. In almost all cases, these transformations yield 1-aminonorbornanes 2. Notably, it is also found that the role of the nitro motif was necessary. For example, a 3,5bis(trifluoromethyl)benzimine system was unable to give the corresponding product upon irradiation at $390 \mathrm{~nm}$ or at shorter wavelengths.

The same strategy has been studied in intermolecular version via a formal [3+2] cycloaddition (Scheme 2). ${ }^{21}$ In this case, cyclopropylimines 8 and substituted alkenes 9 have been used to generate highly functionalized cyclopentylamines 11 . It should be pointed out that in this transformation a [3+2] cycloaddition, hydrolysis of the imine $\mathbf{1 0}$ and $\mathbf{N}$-functionalization (11) are all carried out in a one-pot procedure.

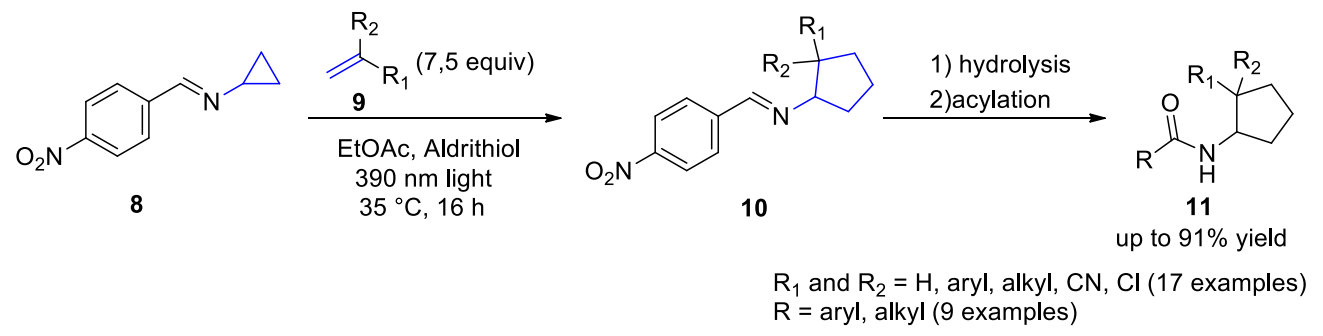


Scheme 2: Photochemically generated masked N-centered radical strategy for the synthesis of cyclopentylamines.

The ring opening of the cyclobutane was also observed under similar conditions (Scheme 3). ${ }^{22}$ Irradiation of the bicyclo [1.1.1] pentan-1-amine derived imine $\mathbf{1 2}$ generates the $\mathrm{N}$-centered radical species 16, which initiates cyclobutane ring cleavage generating the primary radical intermediate 17 . This nucleophilic radical reacts then with an alkene to give an inert bicyclo [3.1.1] heptan-1-imine 14. The reaction has been performed with a variety of styrenes 13 . The imine products are easily hydrolyzed to give primary amine building blocks.

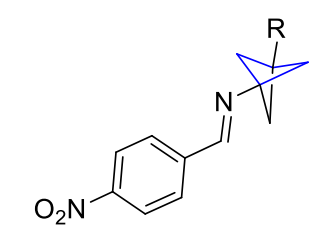

12

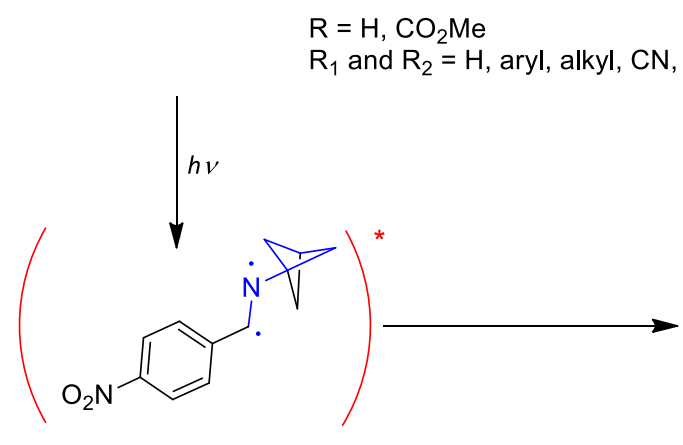

16

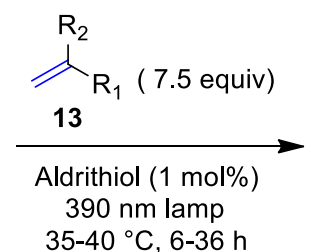

$390 \mathrm{~nm}$ lamp
$35-40{ }^{\circ} \mathrm{C}, 6-36 \mathrm{~h}$

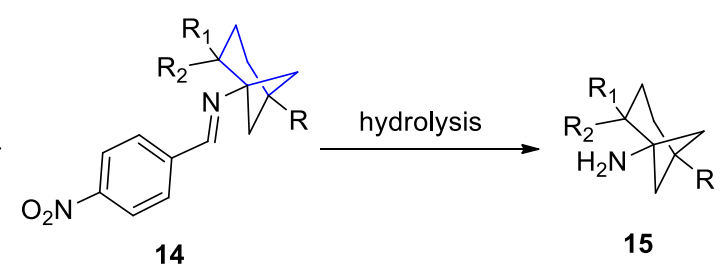

11 examples up to $73 \%$ yield<smiles>[R]C(=C)CCCCC</smiles><smiles>CCC(CC)N=Cc1ccc([N+](=O)[O-])cc1</smiles>

17

Scheme 3: Photochemical synthesis of bicyclo[3.1.1]heptan-1-amines.

The photochemical cyclopropylimine-pyrroline rearrangement has been reported for the first time in $2001 .^{19}$ In 2005, a thorough study of the mechanism, regiochemistry and stereochemistry of the reaction has been published. It was found that electronically excited nonequilibrated diradicals are involved in the mechanism as transients. Their decay leads to pyrrolines and cyclopropylimine isomers. The regiochemistry of the reaction is related to the ability of different groups to stabilize the radical formed during the reaction. The stereochemistry of reaction depends on the irradiation time and the substitution of the imine. Simple and complex substitution pattern of the cyclopropane ring and in the iminic position of the $\mathrm{N}$-cyclopropylimine structure have been used to synthesize the corresponding 1pyrrolines (Scheme 4). ${ }^{20}$ 

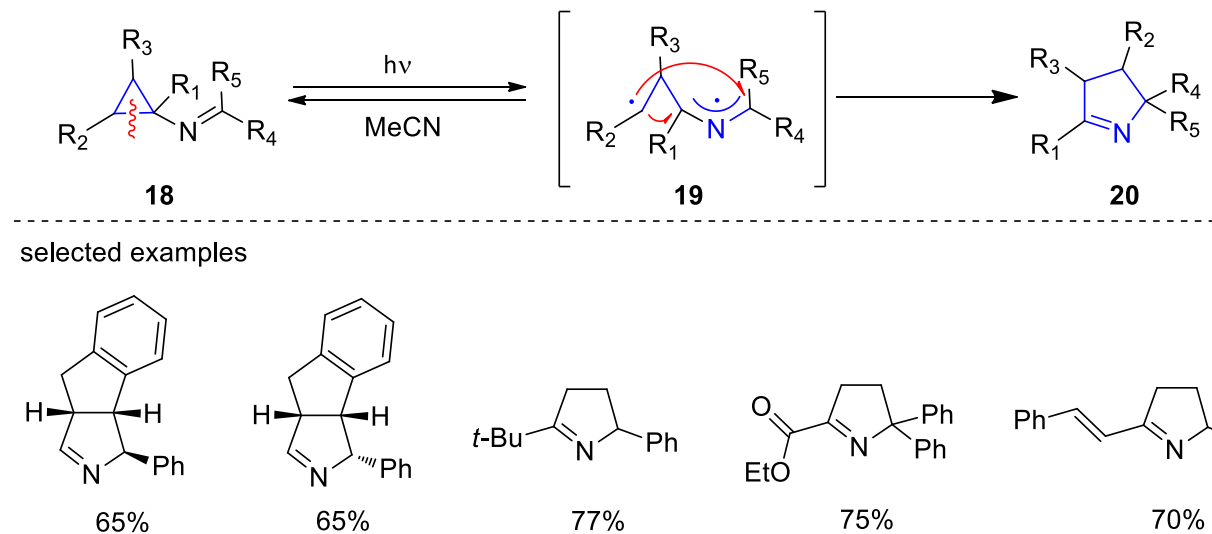

xamples

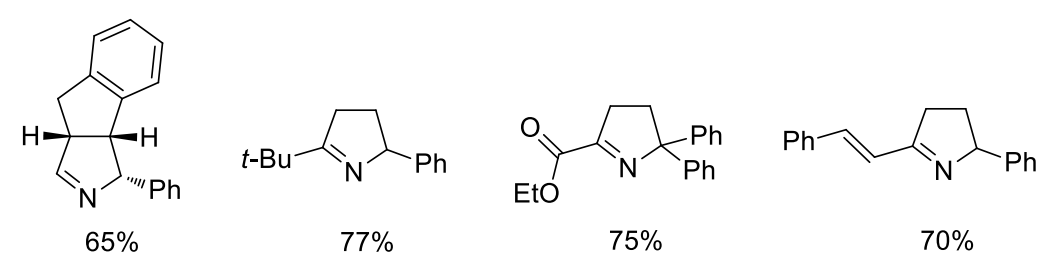

Scheme 4: The N-Cyclopropylimine-1-pyrroline photorearrangement.

In 2015, Kutateladze et al. reported an imine-based photoassisted intramolecular cycloaddition to synthesize complex nitrogen poly-heterocycles (Scheme 5). Irradiation of imines containing $o$-amido groups 21 produces reactive amino azaxylylenes intermediates 22. They are photogenerated via excited state intramolecular proton transfer (ESIPT), which undergo intramolecular [4 + 2] (24) and [4 + 4] (23) cycloadditions to yield complex $\mathrm{N}, \mathrm{O}$-heterocycles with a spiro-connected nitrogen heterocyclic moiety. ${ }^{23}$ A variety of cyclic imines containing $o$-amido groups with tethered furan and pyrrole-based unsaturated pendants have been used to access the corresponding [4+2] and [4+4] formal cycloadditions products in good yields.

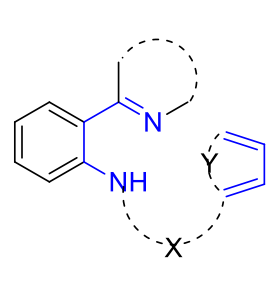

21

19 examples

selected examples

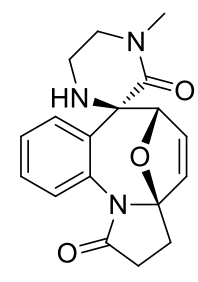

$39 \%$

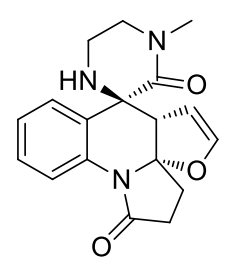

$35 \%$

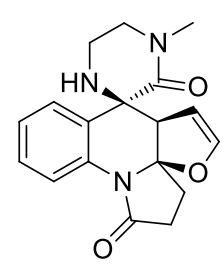

$-$

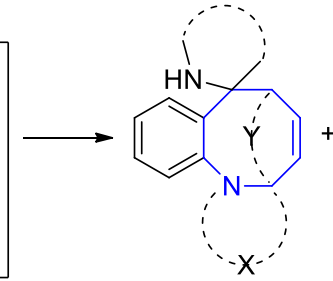

23

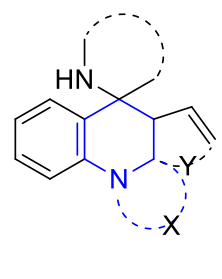

24 amino azaxylylene

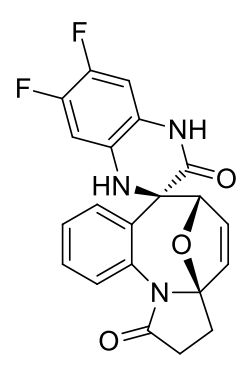

$34 \%$

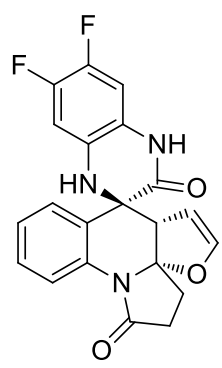

$25 \%$<smiles>O=C1COC23Nc4ccccc4C4(CO)Nc5cc(F)c(F)cc5NC14C2CCO3</smiles>

$11 \%$

Scheme 5: Photogeneration of amino azaxylylenes and their intramolecular photocyclizations. 
The mechanism of such reactions has previously been experimentally and computationally studied with azaxylylenes photogenerated by ESIPT in aromatic 0 -amido ketones and aldehydes bearing unsaturated functionalities (Scheme 6). ${ }^{24}$ Amides of aromatic $\boldsymbol{o}$-ketones and aldehydes $\mathbf{2 5}$ undergo fast and efficient ESIPT leading to their tautomeric form, azaxylylene 26S. Intersystem crossing yields the triplet azaxylylene diradicaloid species $\mathbf{2 6 T}$. In a first step, the nitrogen centered radial adds to a double bond to form the diradical intermediate 27. Radical combination leads to both $\mathbf{2 8}$ and $\mathbf{2 9}$.

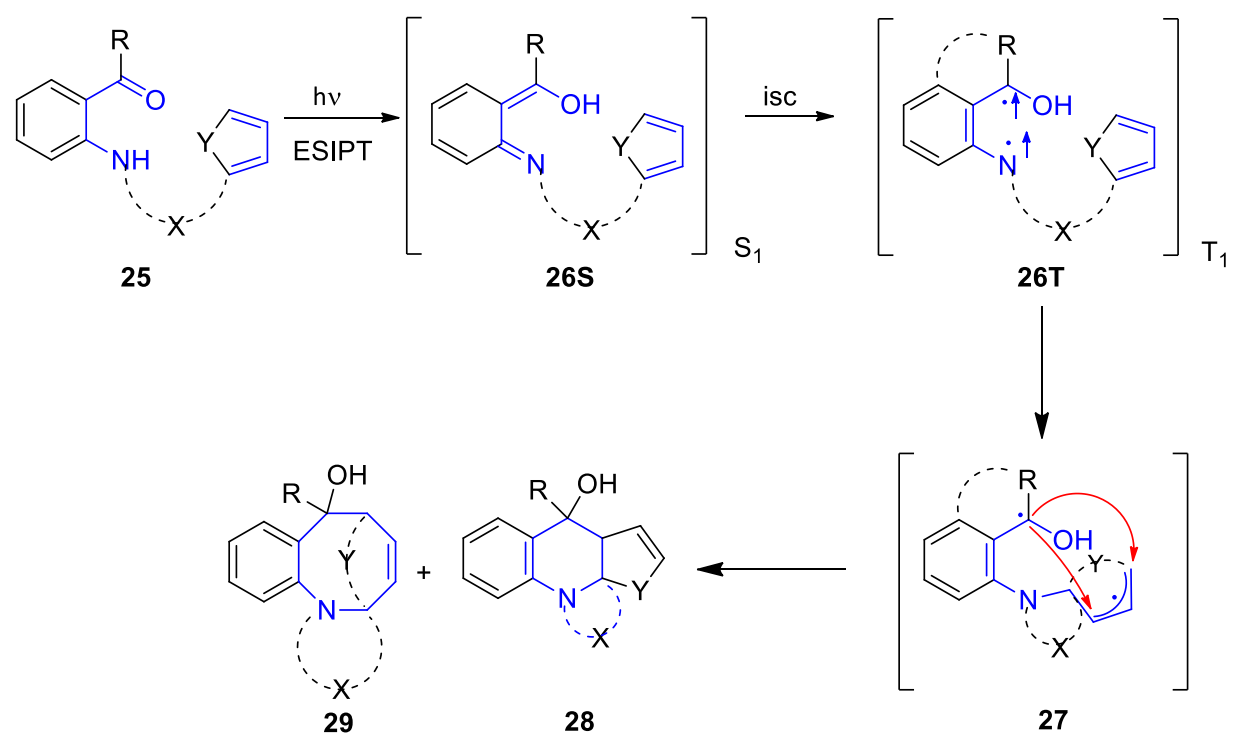

Scheme 6: The proposed mechanism of the intramolecular [4+2] and [4+4] formal cycloadditions.

Hydrogen atom transfer, induced by light absorption, generates high energy radical species that can rearrange or react with other species to form complex structures. Hoffmann et al. reported a photochemically induced intramolecular hydrogen atom transfer in cyclic imines followed by a cyclization leading to two compound families resulting either from the formation of a $\mathrm{C}-\mathrm{C}$ bond or a $\mathrm{C}-\mathrm{N}$ bond (Scheme 7). ${ }^{25} \mathrm{~A}$ mechanism supported by a computational study is suggested. After electronic excitation of imine function $\mathbf{3 0}$ which generates the triplet excited state diyl $\mathbf{3 4}$, an intramolecular hydrogen atom transfer occurs from the acetal moiety to the imine nitrogen atom in one-step process (the proton and the electron are simultaneously transferred). Single electron transfer from acetal functions is very difficult ${ }^{26}$ which excludes a two-step HAT process (the electron is transferred first, and the proton follows). ${ }^{27}$ The hydrogen abstraction at the carbon atom of the imine function is energetically less favored. The resulting diradical $\mathbf{3 5}$ undergoes intersystem crossing to give singlet state intermediate 36 or 36'. C-C Coupling reaction yields the bicyclo[3.2.1]-octane derivative $\mathbf{3 2}$ and the N-C coupling produces the bicyclo[3.3.0]octane derivative 31. Similar reactions have been carried out with $\alpha, \beta$ unsaturated furanones. ${ }^{28}$ 


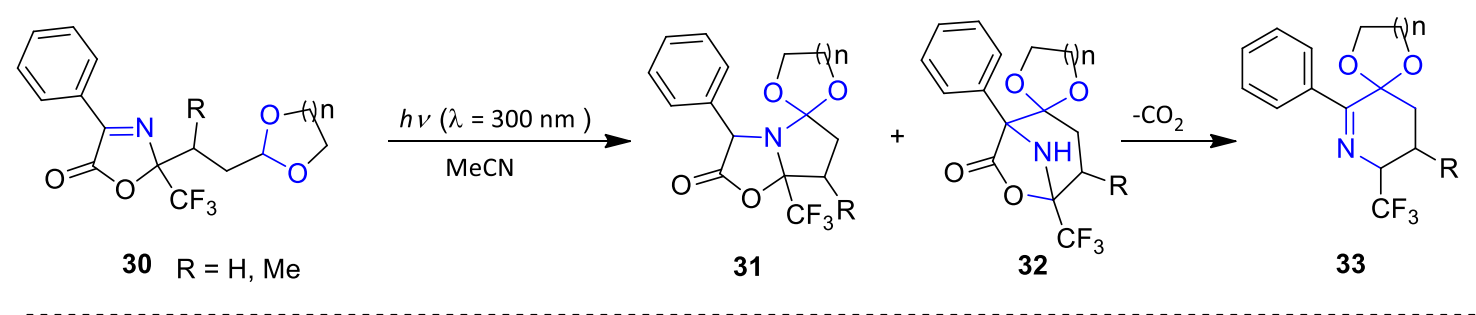

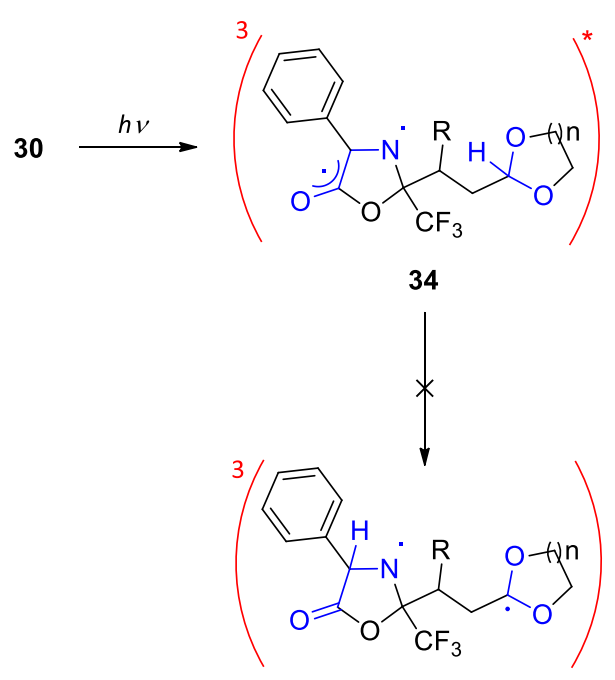

37

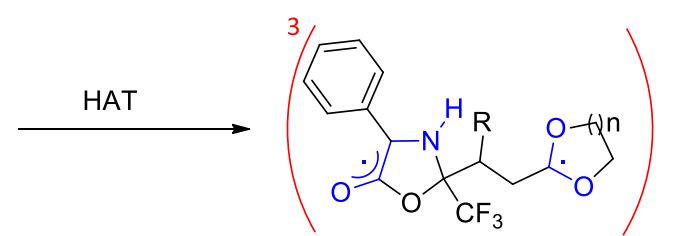

35

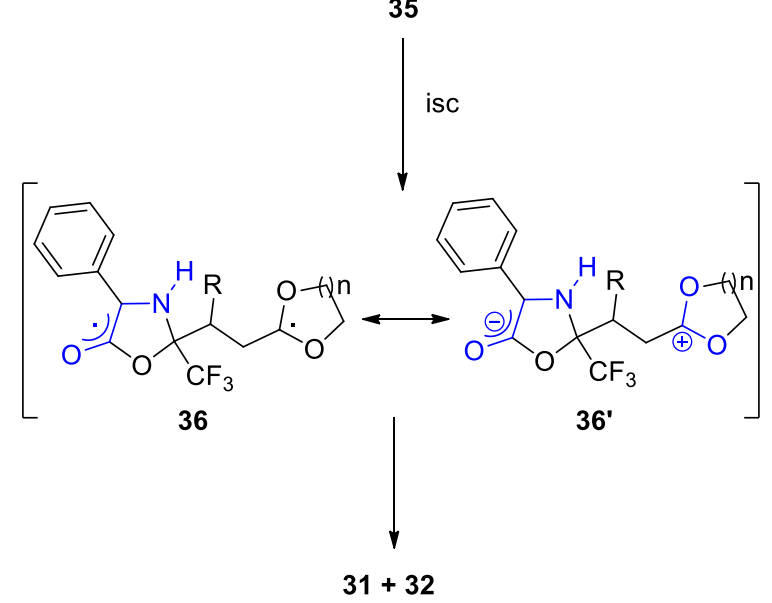

Scheme 7: Photoinduced intramolecular radical cyclisation via 1,5-HAT with imines.

\subsection{Generation of radical intermediates by photoredox catalysis.}

Numerous reports of radical additions to imines under visible light photocatalysis for the synthesis of saturated N-heterocycles have been reported. In 2016, Bode's group described a photocatalytic synthesis of piperazines from silicon amine protocol (SLAP) reagents with an imine moiety using [Ir(ppy)2dtbbpy]PF6 as the catalyst (Scheme 8$).{ }^{29}$ Single-electron oxidation of the $\alpha$-silyl amine $\mathbf{3 8}$ by photoexcited $\operatorname{Ir}(\mathrm{III})$ * complex and desilylation generate the $\operatorname{Ir}(\mathrm{II})$ species and the nitrogen stabilized carbon-centered radical 40. This latter species adds to iminic carbon giving the cyclized $\mathrm{N}$-centered radical 41. A single-electron-transfer reduction followed by protonation affords the piperazine product 39 and regenerates the $\mathrm{Ir}(\mathrm{III})$ catalyst. This reaction tolerates a wide range of heteroaromatic, aromatic, and aliphatic SLAP reagents. 


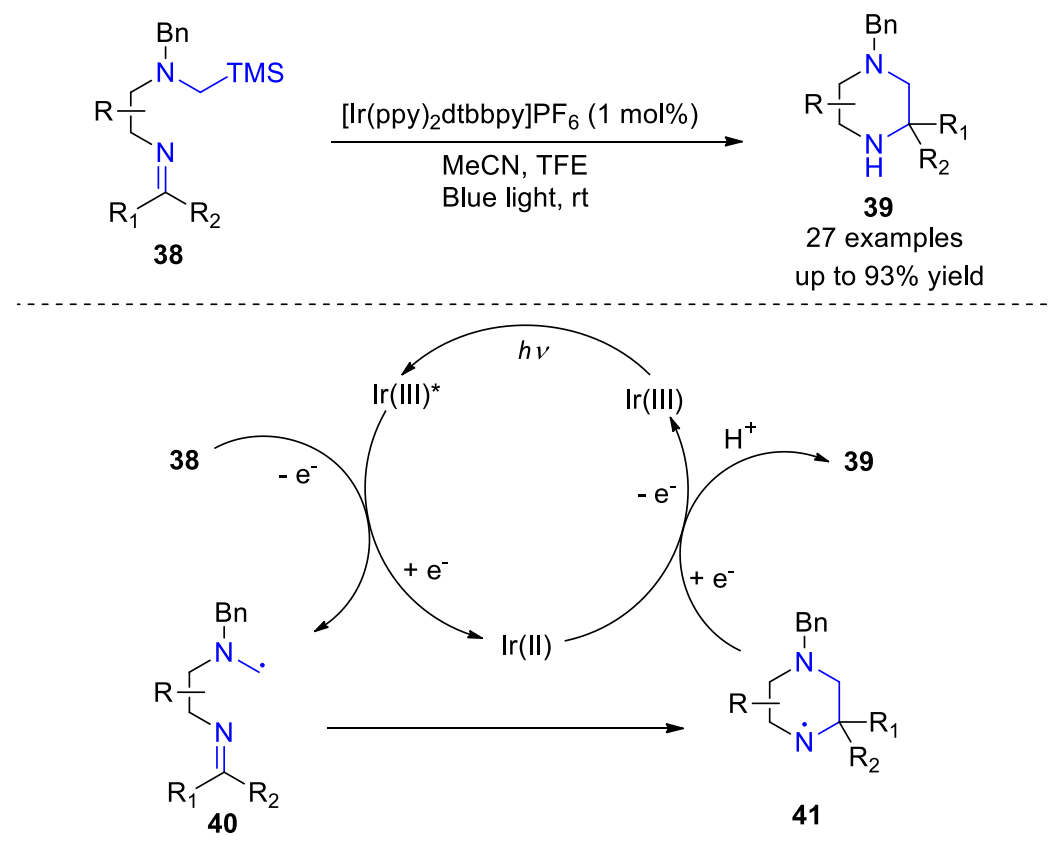

Scheme 8: Photoredox catalytic synthesis of piperazines using SLAP reagents and the proposed mechanism.

The synthesis of substituted morpholines, oxazepanes, thiomorpholines, and thiazepanes (43) has also been reported (Scheme 9). ${ }^{30}$ The key point in this transformation is the use of a Lewis acid to activate the imine $\mathbf{4 2}$ which induces an alternative photocatalytic cycle with an initial Lewis acid-assisted singleelectron reduction of the imine.

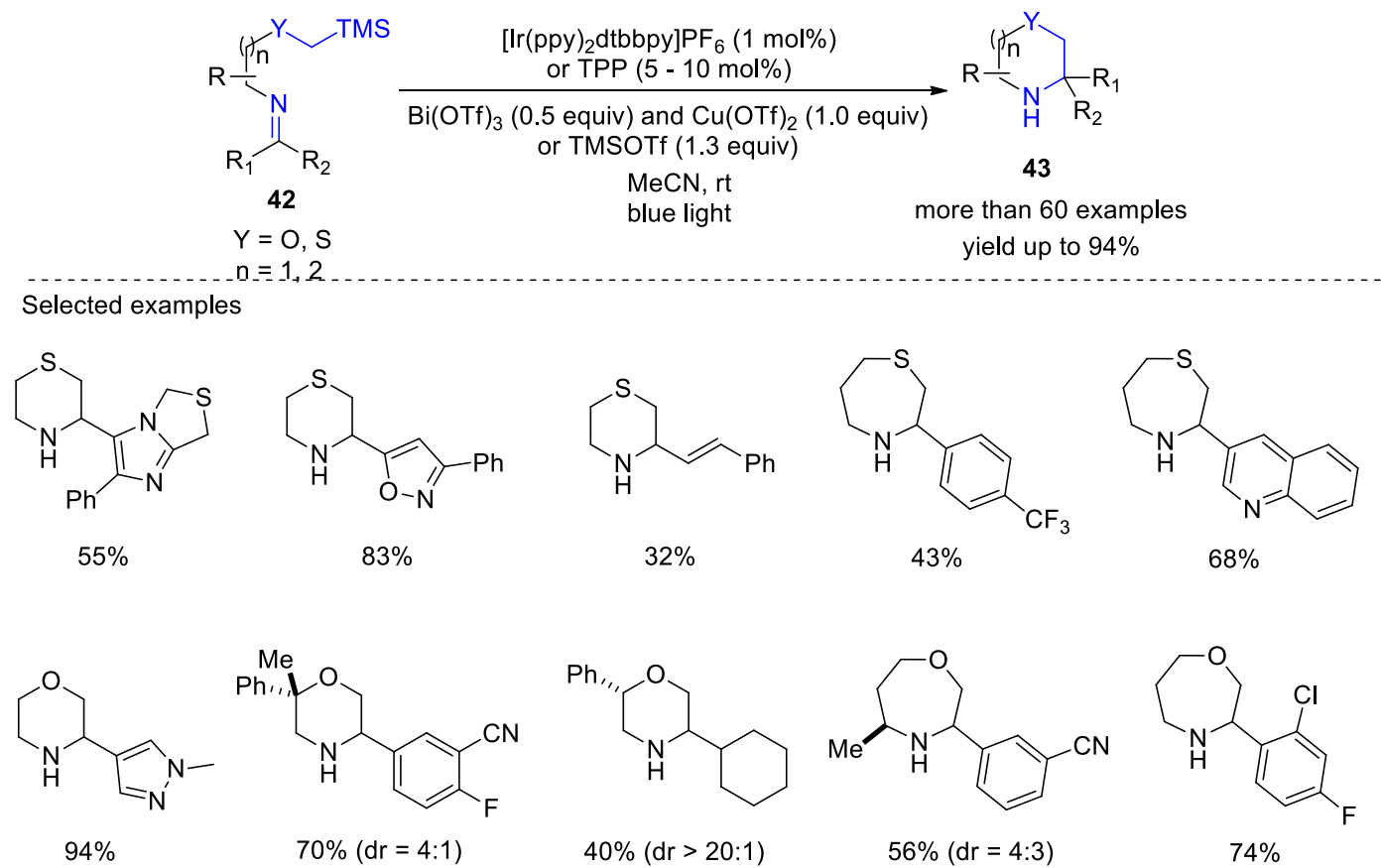


Scheme 9: Photoredox catalytic synthesis of saturated N-heterocycles using SLAP reagents.

Shortly thereafter, Romanov-Michailidis et al. also developed an efficient approach for the synthesis of saturated nitrogen containing heterocycles using a dual-catalyst system composed of an iridium photocatalyst and a lithium phosphate base (Scheme 10).31 Condensation of complex aminodihydropyridine (DHP) reagents 44 with heteroaromatic, aromatic, and aliphatic aldehydes 45 give imino-tethered DHP intermediates 46 that undergo photoredox catalytic cyclization with visible light irradiation to afford corresponding cyclization products 47 in high yields (54-86\%) and with excellent levels of diastereocontrol (up to $>14: 1 \mathrm{dr}$ ). The mechanism begins with a proton coupled electron transfer (PCET) quenching of the photoexcited $\operatorname{Ir}(I I I) *$ species by imino-DHP 46 with help from the Brønsted-basic phosphate anion (X-)32 to give radical-cationic intermediate 48 which then undergoes $\beta$ cleavage to affords $\alpha$-heteroatom stabilized $\mathrm{C}$-centered radical 49 . The latter adds to the iminic carbon via 6-endo-trig cyclization to give $\mathrm{N}$-centered radical 50 . Single-electron-transfer reduction followed by proton transfer leads to the product and regenerates $\operatorname{Ir}($ III) photocatalyst.
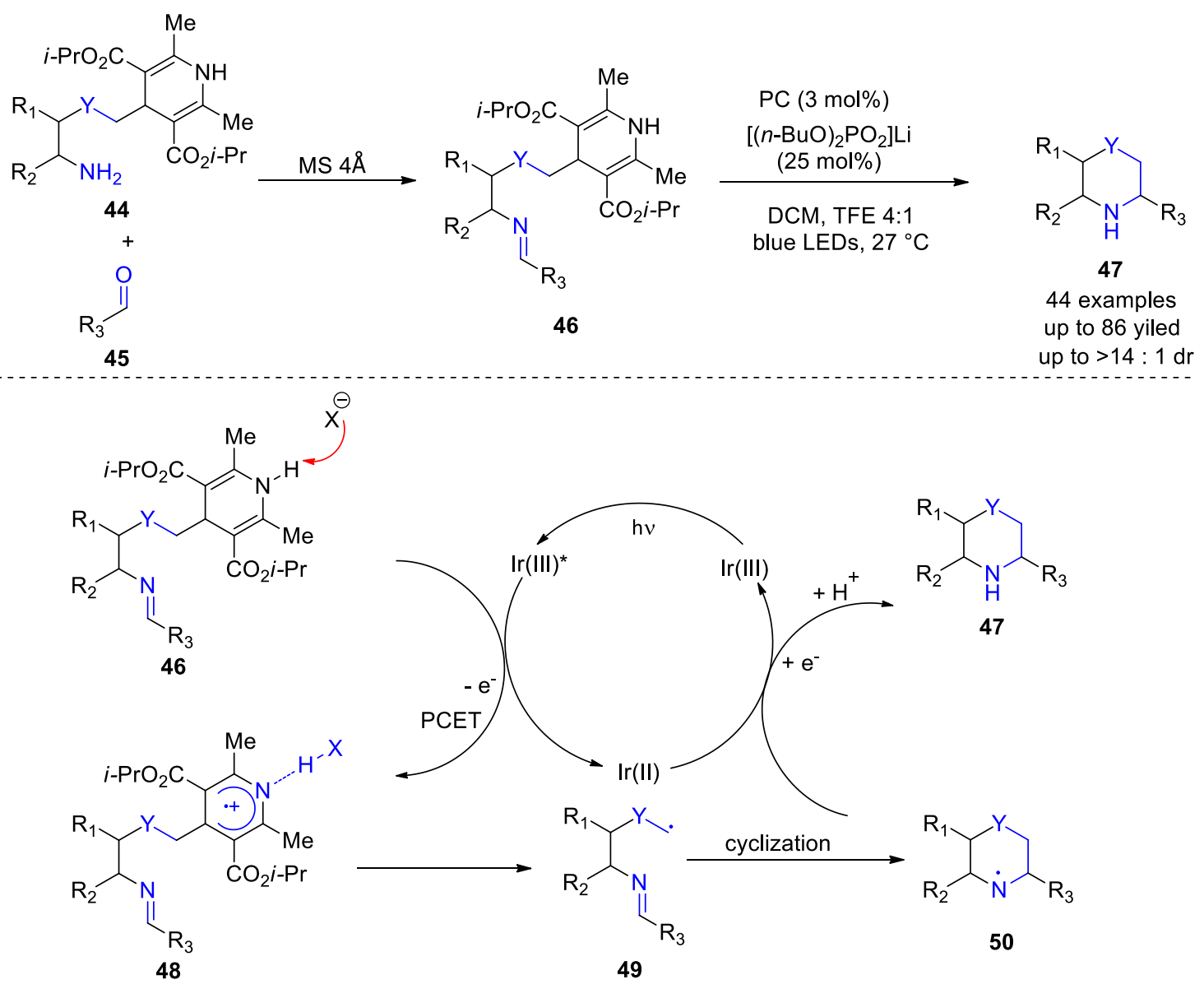

Scheme 10: Synthesis of saturated N-heterocycles by photoredox cyclization of imino-tethered dihydropyridines and the proposed mechanism. 
Radical alkylation and subsequent cyclization in photoredox-mediated radical/polar crossover (RPC) process leading to saturated $\mathrm{N}$-heterocycles has been reported (Scheme 11). ${ }^{33}$ Under visible light irradiation conditions, alkyl radicals possessing pendant leaving groups (e.g. bromide) generated from corresponding alkyl silicates leads to $\mathrm{C}-\mathrm{C}$ bond formation with imines. Subsequent reduction of the resulting nitrogen-centered radical $\mathbf{5 4}$ furnishes an amide anion $\mathbf{5 5}$ that engage the leaving group in an intramolecular nucleophilic substitution. The substituted pyrrolidines, piperidines, and azepanes were obtained in yields up to $84 \%$. Further examples of radical reactions with silicates have been published. ${ }^{34}$

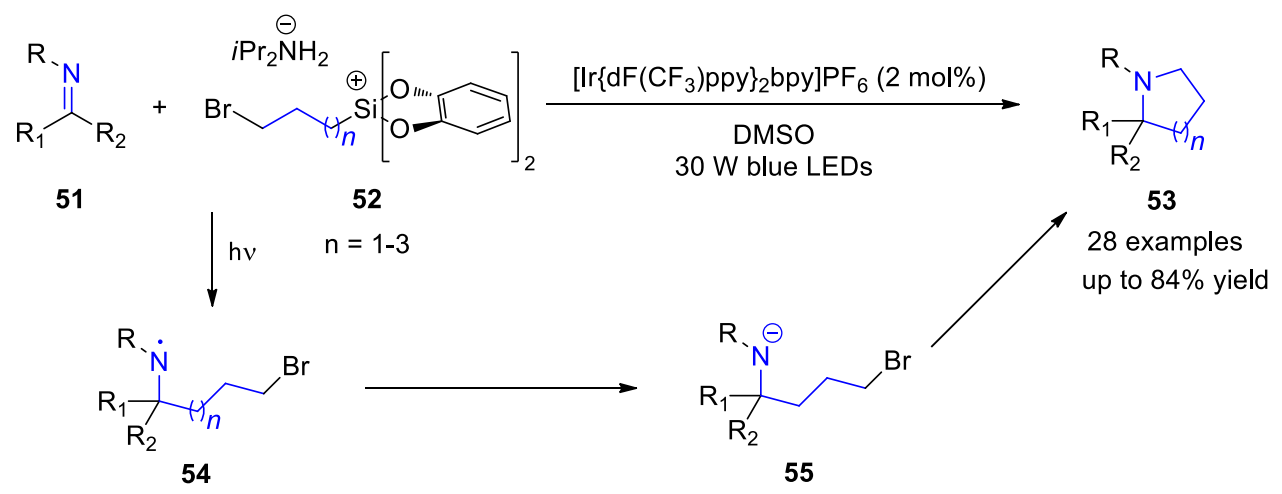

Scheme 11: Photoredox-mediated radical/polar crossover (RPC) process for the synthesis of saturated N-heterocycles.

In 2014, the group of Wu and Liu developed a visible-light supported method for aerobic catalytic oxidative cyclization of phenol-2-imines 56 (Scheme 12). ${ }^{35}$ By combining bis(tetrabutylammonium salt) (TBA)-eosin $\mathrm{Y}$ and molecular oxygen as oxidant in the presence of 1,8-diazabicyclo[5.4.0]undec-7-ene (DBU) as a base, 39 examples of phenol-2-imines $(56)+$ were readily transformed with excellent yield into 2 -substituted benzoxazoles $\mathbf{5 7}$. The suggested photocatalytic aerobic dehydrogenation mechanism is depicted in Scheme $12 .{ }^{36}$ The phenol moiety can be easily deprotonated to the corresponding phenolate anion 58, a much stronger electron donor, in the presence of DBU. The efficient electron transfer from phenolate anion to the excited TBA-eosin Y generates the phenoxyl radical 59. The photocatalyst is regenerated by oxidation of the TBA-eosin $\mathrm{Y}$ radical ion by molecular oxygen. The phenoxyl radical $\mathbf{5 9}$ undergoes intramolecular 5-endo cycloaddition to the imine carbon atom to form the corresponding aminyl radical 60 which can react with the photocatalyst or superoxide radical anions to afford the dehydrogenated product $\mathbf{5 7}$ selectively. 


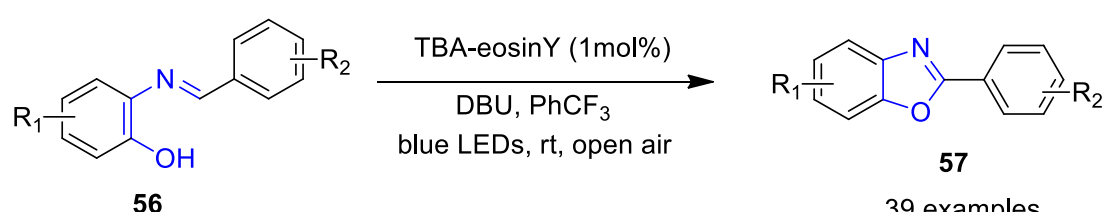

56

39 examples $61-96 \%$ yield

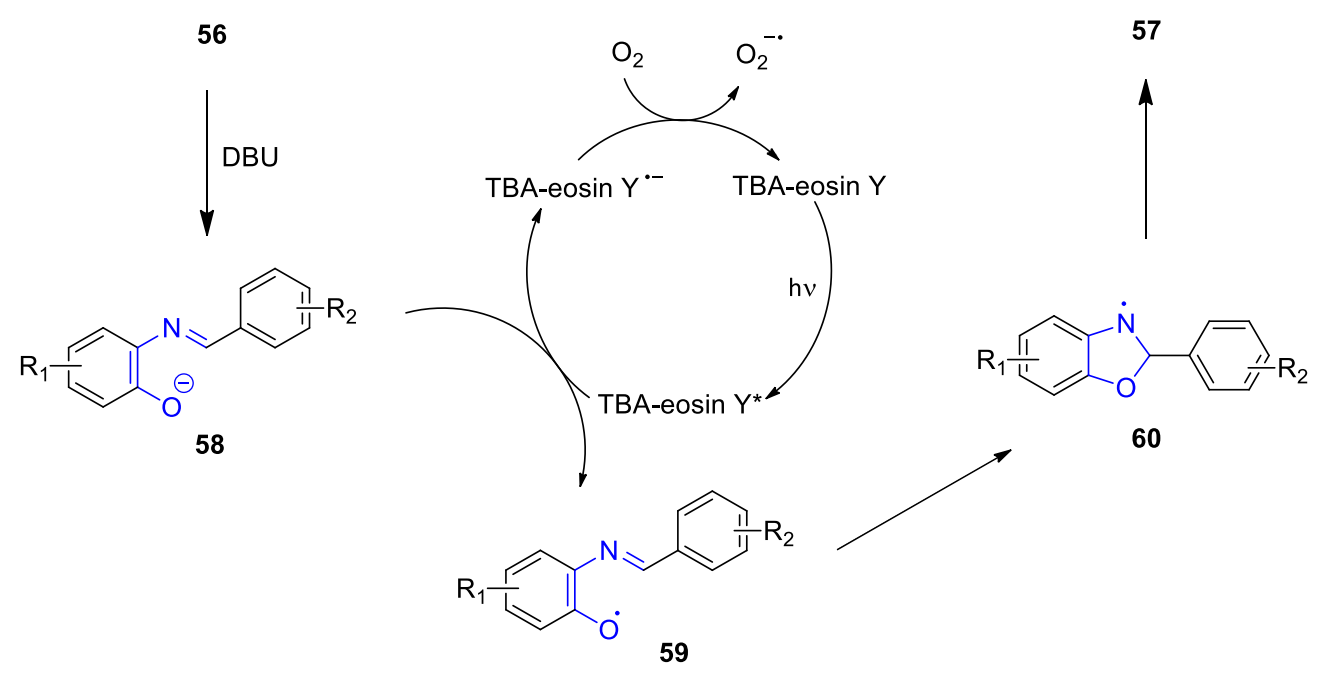

Scheme 12: Visible-light supported aerobic catalytic oxidative cyclization of 2-substituted phenolic imines and the proposed mechanism.

The photocycloaddition reaction of $2 \mathrm{H}$-azirines, known for a long time, is an extremely versatile and important process for the synthesis of aza-heterocycles. Remarkable achievements have been made toward understanding the factors which determine the photochemical behavior in a given system. ${ }^{37}$ In 1991, Müller and Mattay reported the synthesis of imidazolophanes 62 and pyrrolophanes 63 by means of photoinduced electron transfer (PET) using 1, 4-naphthalenedicarbonitrile (DCN) as the photocatalyst (Scheme 13). ${ }^{38}$ Under these conditions, the electron acceptor is excited by irradiation with light at $\lambda=350 \mathrm{~nm}$ in the presence of azirine $\mathbf{6 1}$, leading to the $D C N$ radical anion and a reactive azirine radical cation. This latter species opens to the linear 2-azaallenyl radical cation 64 . In a two-step process, radical addition of $\mathbf{6 4}$ to activated alkynes or imine double bond leads to intermediates 65,66 which subsequently undergo a reduction/cyclization sequence $(67,68)$ followed by aromatization to give corresponding imidazolophanes 62 and pyrrolophanes 63. 

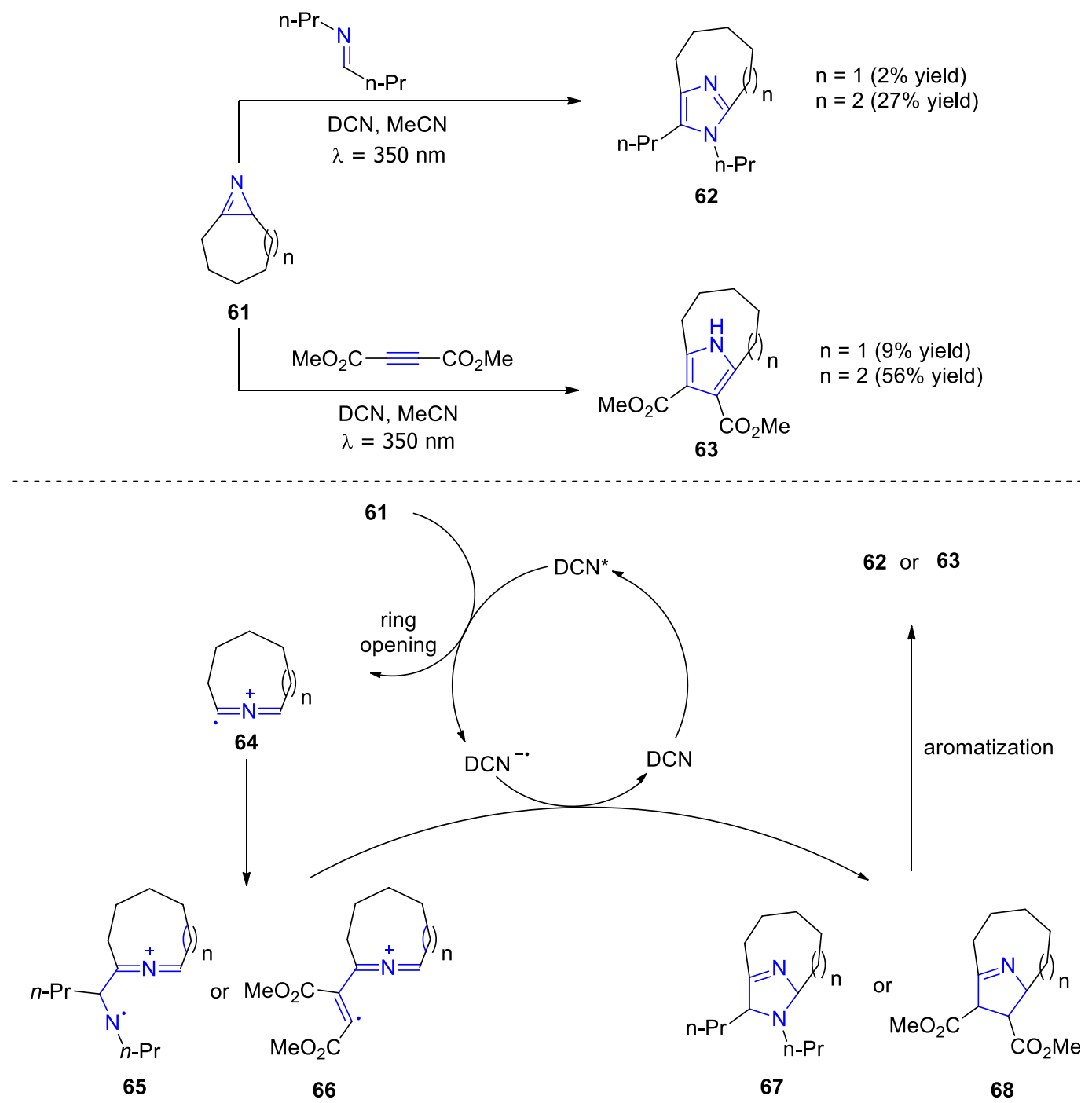

Scheme 13: The synthesis of imidazolophanes and pyrrolophanes.

Recently, Xiao and Lu et al. reported a visible light photoredox-catalyzed [3 + 2] cycloaddition/oxidative aromatization sequence of $2 \mathrm{H}$-azirines 69 and aldehydes $\mathbf{7 0}$ using 9-mesityl-10-methylacridinium perchlorate as a photocatalyst and 2,3-dichloro- 5,6-dicyano-1,4-benzoquinone (DDQ) as an oxidizing agent (Scheme 14). ${ }^{39}$ This catalytic system provides an efficient access to highly functionalized oxazoles 71 from easily accessible $2 \mathrm{H}$-azirines and aldehydes. The excited photocatalyst first oxidizes the $2 \mathrm{H}$ Azirine 69 by a single-electron-transfer process to give radical cation $\mathbf{7 2}$ which then undergoes homolytic cleavage of the $\mathrm{C}-\mathrm{C}$ bond to generate the 2-azaallenyl radical cation 73. Nucleophilic attack of benzaldehyde on $\mathbf{7 3}$ delivers the intermediate $\mathbf{7 4}$, which subsequently undergoes an intramolecular radical cyclization to afford the new radical cation $\mathbf{7 5}$. After that, this transient species $\mathbf{7 5}$ undergoes another single-electron-transfer reduction by the reducing photocatalyst to afford the cycloadduct $\mathbf{7 6}$ with the concomitant regeneration of photocatalyst. Finally, the oxidative aromatization of 2,5- 
dihydrooxazole 76 yields the desired oxazole product 71. Under similar conditions, electro-deficient alkynes react with $2 \mathrm{H}$-azirines to yield polysubstituted pyrroles. ${ }^{40}$

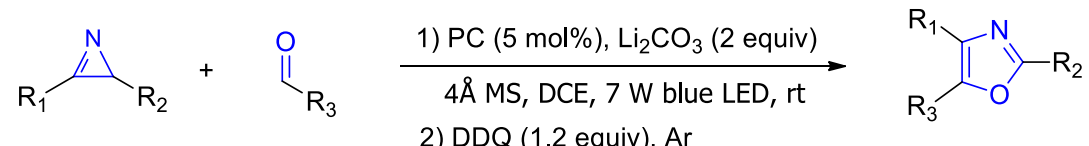

69

70
71

20 examples up to $80 \%$ yield

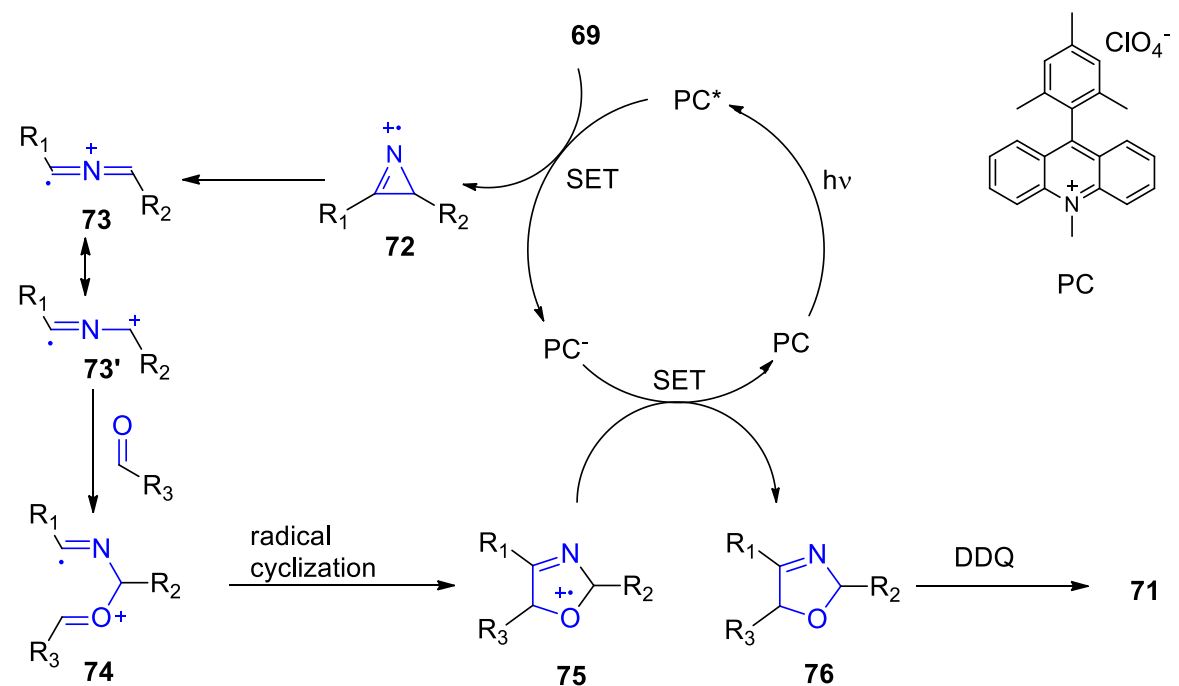

Scheme 14: Visible light photoredox-catalyzed [3 + 2] cycloaddition/ oxidative aromatization sequence of $2 \mathrm{H}$-azirines and aldehydes.

Further studies of consecutive radical addition cyclization reactions leading to N-polyheterocyclic compounds have been reported. In 2018, Dixon's group described a visible light photocatalyzed polarity reversed Povarov reaction of aryl imines $\mathbf{7 7}$ and electron deficient alkenes. Using [Ir\{dF(CF $\left.\left.\mathrm{CF}_{3}\right) \mathrm{ppy}_{2}(\mathrm{dtbpy})\right] \mathrm{PF}_{6}$ as photocatalyst and the commercial Hantzsch ester as a stoichiometric reductant, a range of substituted tetrahydroquinoline $\mathbf{7 9}$ were obtained in high yields and with typically good to excellent diastereoselectivity in favor of the trans diastereoisomer (Scheme 15). ${ }^{41} \mathrm{~A}$ proton coupled electron transfer (PCET) based mechanism is proposed. After photochemical excitation of the photo-catalyst, electron transfer occurs from the Hantzsch ester (HE) derivative to the catalyst yields the $\operatorname{Ir}(I I)$ species. Electron transfer from this $\operatorname{Ir}(I I)$ species coupled to a proton transfer from the Hantzsch ester radical cation to the imine function leads to the $\alpha$-amino radical $\mathbf{8 1}$ which then reacts with the phenyl vinyl sulfone in a step-wise radical cyclization to give the stabilized Povarov radical intermediate 82. In a base assisted homolytic aromatic substitution-type mechanism, this intermediate $\mathbf{8 2}$ loses a proton to form strongly reducing radical anion intermediate $\mathbf{8 3}$ which can reduce fresh imine substrate $\mathbf{8 0}$ with concomitant protonation to lead to the product $\mathbf{8 4}$ and regenerates the key $\alpha$-amino radical in a radical chain process. 


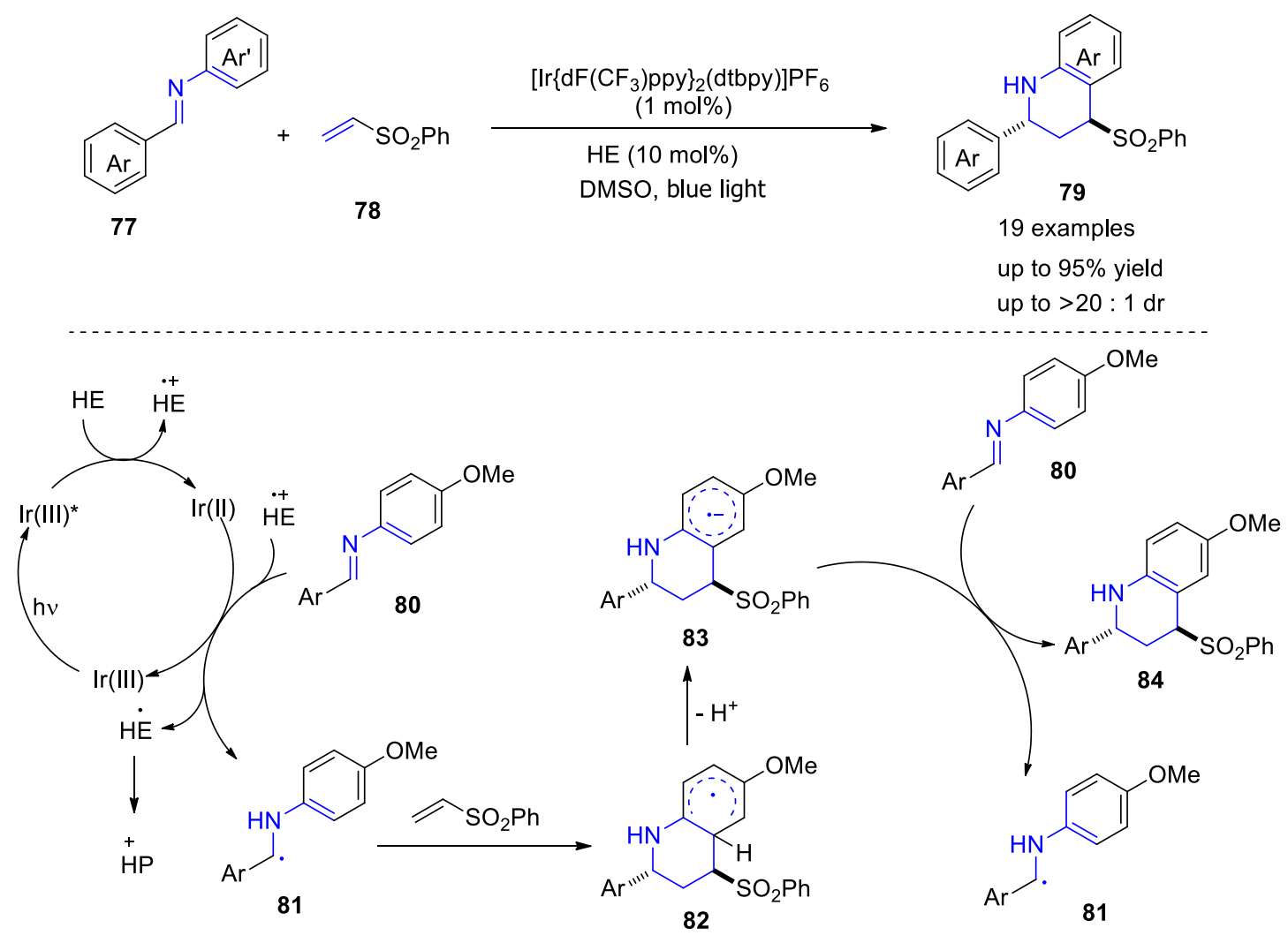

HE: Hantzsch ester

HP: Hantzsch pyridine

Scheme 15: The photocatalytic polarity reversed Povarov reaction and the postulated mechanism.

Another consecutive radical addition cyclization reaction leading to various isoquinolines and related polyaromatics has been reported (Scheme 16). ${ }^{42}$ In a photoredox catalytic process, an iminyl radical intermediate $\mathbf{8 8}$ is generated by $\mathrm{N}-\mathrm{H}$ cleavage. The latter initiates a cascade $\mathrm{C}-\mathrm{N} / \mathrm{C}-\mathrm{C}$ bonds formation in the presence of alkynes to give the cyclization products 87 in good yields up to $92 \%$. It should be noted that the photocatalyst is regenerated in a cobalt co-catalytic cycle. In these steps the intermediate $\mathbf{9 0}$ is transformed into the final product $\mathbf{8 7}$ (rearomatization) via electron and proton transfer to the $\mathrm{Co}$ (II) species.

Using a dual photoredox/cobaloxime catalytic system, oxidative [4+2] annulation of $\mathrm{NH}$ imines and alkenes for the synthesis of multisubstituted 3,4-dihydroisoquinolines has been described. ${ }^{43}$ 

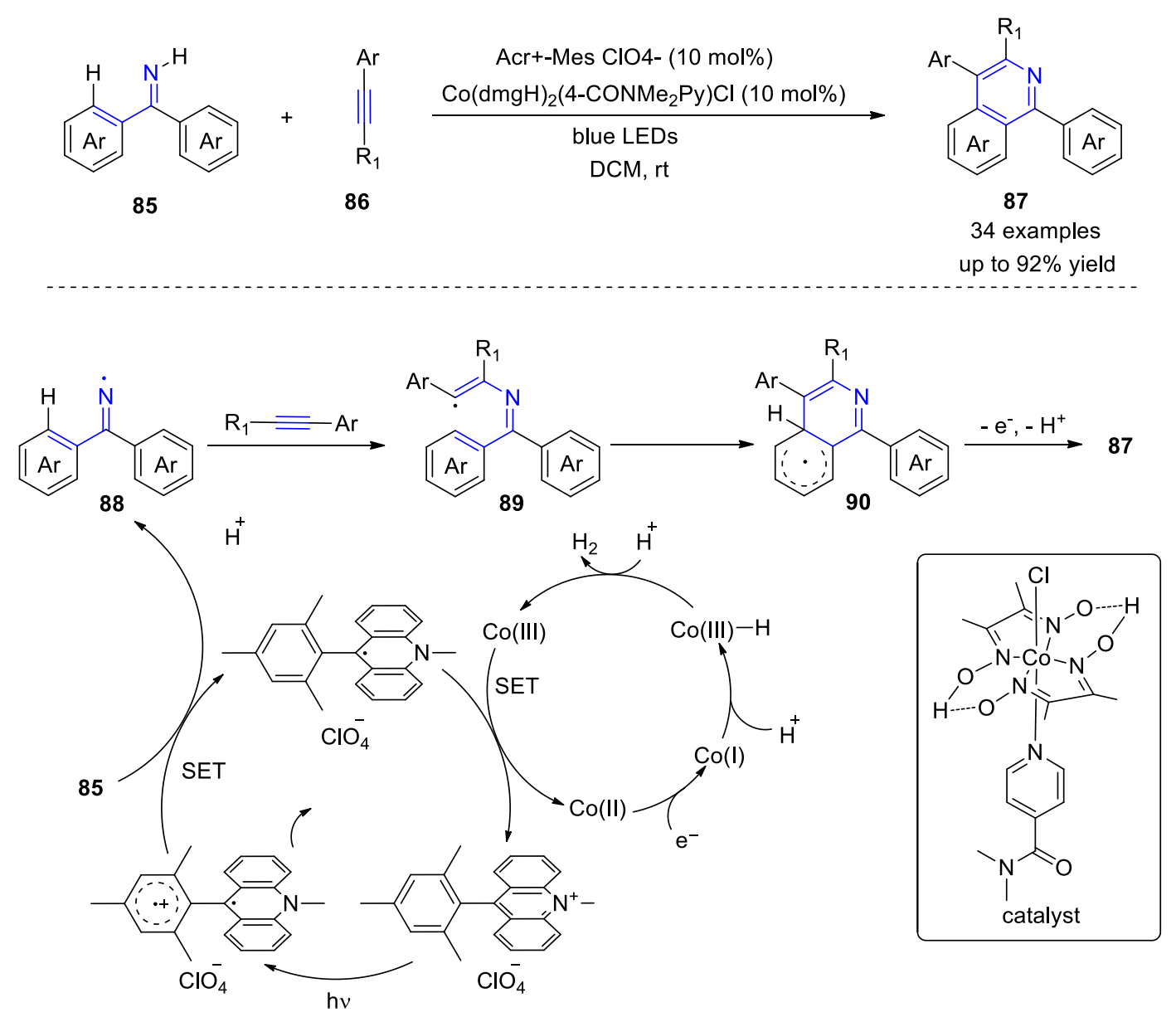

Scheme 16: Visible-light synthesis of isoquinolines and related polyaromatics.

\section{3 imidoyl chlorides}

Activation of C-heteroatom bond on the imine carbon is useful for the generation of imidoyl radicals by photoredox catalysis. This attractive intermediate has been used as key intermediate in the synthesis of various nitrogen-containing heterocycles. Intra- and intermolecular additions of imidoyl radicals to triple carbon-carbon and carbon-nitrogen bonds, as well as cyclizations onto aromatic rings under visible-light irradiation were reported. ${ }^{44}$ In 2013, Zhou et al. reported a visible-light-mediated radical addition cyclization of trifluoroacetimidoyl chlorides $\mathbf{9 1}$ with alkynes $\mathbf{9 2}$ for the synthesis of 2-trifluoromethyl quinolines 93 using imidoyl radicals as a key intermediate (Scheme 17). ${ }^{45}$ The reaction is initiated by photochemical excitation of $\left[\mathrm{Ru}(\mathrm{bpy})_{3}\right]^{2+}$. Single-electron-transfer from electron donor $(n-\mathrm{Bu})_{3} \mathrm{~N}$ to excited $\left[\mathrm{Ru}(\mathrm{bpy})_{3}\right]^{2+*}$ generates $\left[\mathrm{Ru}(\mathrm{bpy})_{3}\right]^{+}$and the radical cation of tributylamine. The imidoyl chloride is reduced and a chloride ion is released. The resulting radical intermediate $\mathbf{9 4}$ undergoes radical addition (95) cyclization with the alkyne $\mathbf{9 2}$. The bicyclic radical intermediate $\mathbf{9 6}$ is oxidized by either the photoexcited $\left[\mathrm{Ru}(\mathrm{bpy})_{3}\right]^{2+*}$ catalyst or the radical cation $(n-\mathrm{Bu})_{3} \mathrm{~N}^{++}$yielding the cation intermediate 97. After deprotonation (rearomatization), the final product $\mathbf{9 3}$ is obtained. 
Various functional groups including methoxy, ester, and ketone present in alkyne, and a variety of para, meta, ortho-substituted phenylacetylenes all worked well to afford the 2-trifluoromethyl quinolines in good yields.
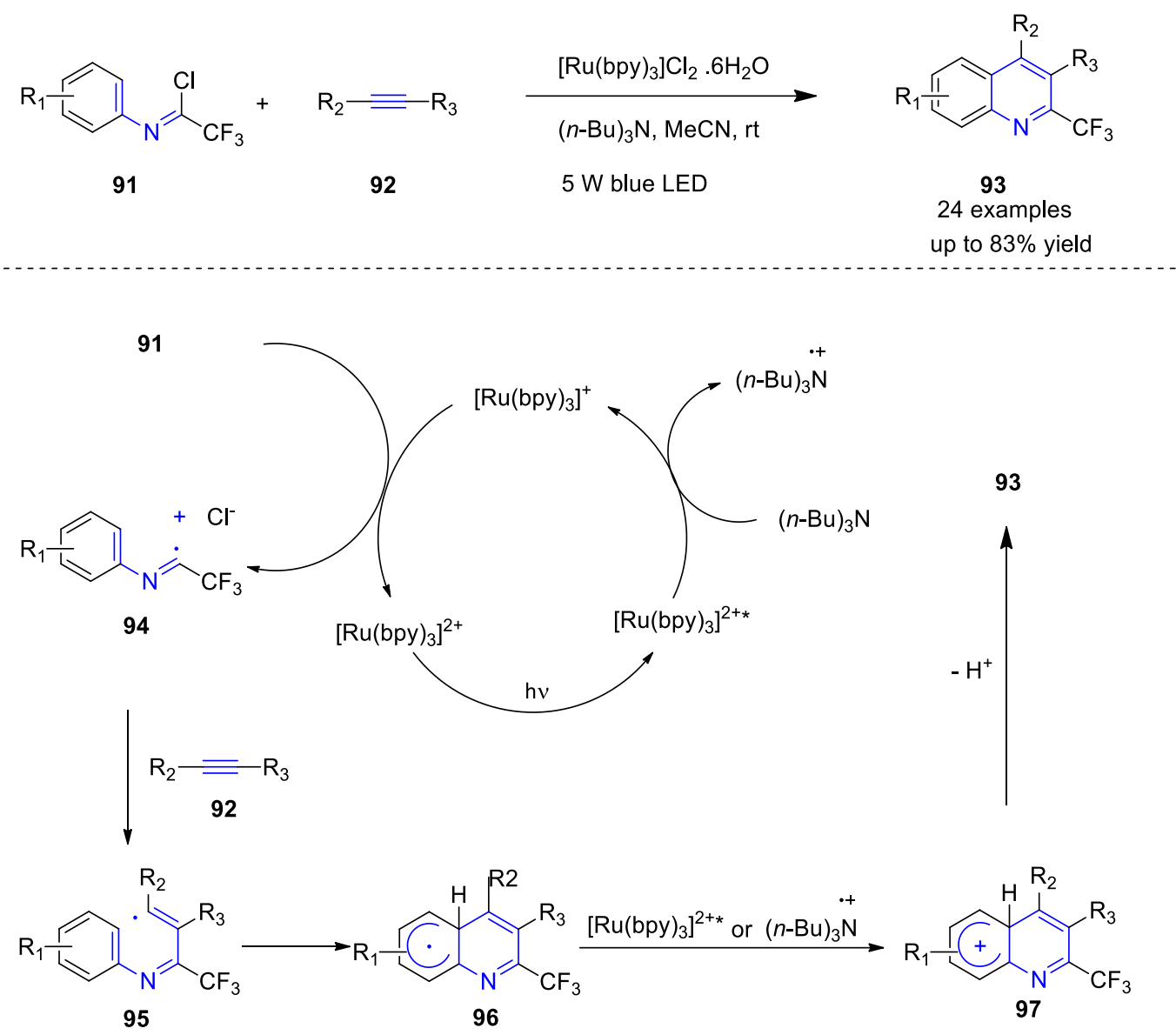

Scheme 17: The synthesis of 2-trifluoromethyl quinolines and the proposed mechanism.

Under similar conditions, the intramolecular cyclization version was also described. In this case, $\mathrm{N}$-[2(alkynyl)phenyl]trifluoroacetimidoyl chlorides $\mathbf{9 8}$ were used to synthesize 2-trifluoromethyl indoles $\mathbf{9 9}$ carrying acyl groups in position 3 (Scheme 18a). A large variety of functional groups can tolerate these mild reaction conditions. ${ }^{46}$

A similar reaction was carried out by Fu et al. for the synthesis of 6-trifluoromethyl phenanthridines 101 through photoredox-catalyzed intramolecular radical cyclization of trifluoroacetimidoyl chlorides $\mathbf{1 0 0}$ (Scheme 18b). ${ }^{47}$ 
a)
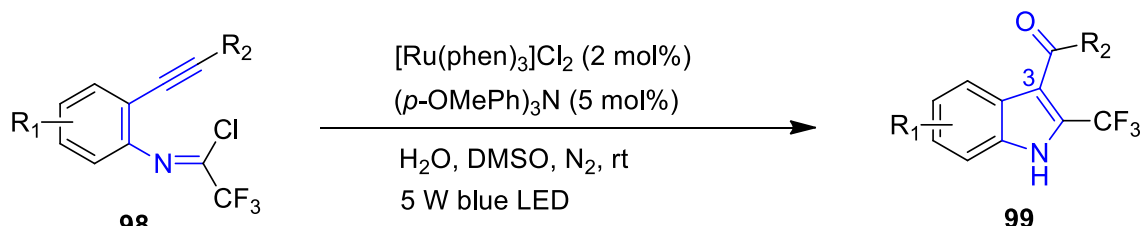

99

18 examples up to $93 \%$ yield

b)<smiles></smiles>

100
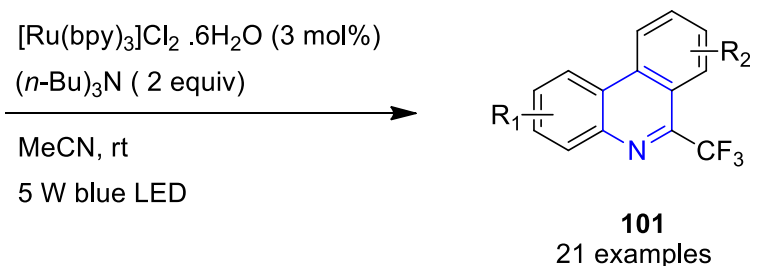

Scheme 18: Light-promoted imidoyl radical cyclization.

\section{Oximes}

\subsection{Generation of radical intermediates by electronic excitation of the substrates}

In photochemical or microwave induced reactions, oximes are suitable precursors for nitrogen centered radicals. ${ }^{48} \mathrm{~A}$ variety of nitrogen containing heterocycles are obtained in this way. A typical example is depicted in Scheme 19. Irradiation of the oxime derivative 102 leads to homolytic fragmentation of the $\mathrm{N}-\mathrm{O}$ bond (103). ${ }^{49}$ Concomitantly formed acyloxyl radicals or phenyl radicals (resulting from decarboxylation) preferentially react with the solvent. Cyclization of intermediate 103 yields the phenanthridine 104. This reaction is frequently observed. For example, it plays a key role in the photochemical stability of pesticides containing this functional group..$^{50}$ In this context, a profound theoretical investigation has been carried out on the competition between the bond fragmentation and $\mathrm{E} / \mathrm{Z}$ isomerization of the oxime function. A series of phenanthridine have been synthesized using sensitization with dicyanoanthracene. ${ }^{51}$ In this case mechanistic studies based on the Hammett plots were carried out.

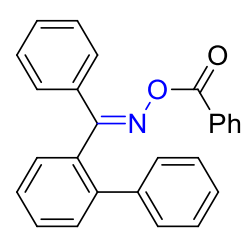

102

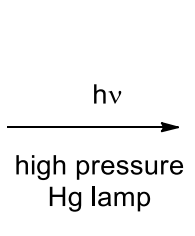

Hg lamp
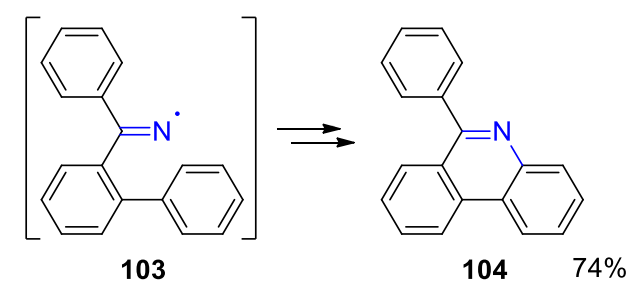

Scheme 19. Photochemically induced radical cyclization via formation of nitrogen centered radical leading to phenanthridine. 
The same reaction was carried out with benzofuran 105a and benzothiophene derivatives 105b (Scheme 20). ${ }^{52}$ In this case, the oxime was used with a carbonate protecting group. The iminyl radical intermediate 106 was generated by UV irradiation. EPR spectroscopy has been carried out. DFT calculations revealed that spirocyclization (107) is kinetically favored, especially at low temperatures, while ortho cyclisation (108) is thermodynamically favored. The corresponding phenanthridines 109a,b have been isolated in good yields. The synthesis strategy can be further extended in heterocyclic chemistry, for example to the preparation of corresponding pyrrolizidine derivatives. ${ }^{53}$

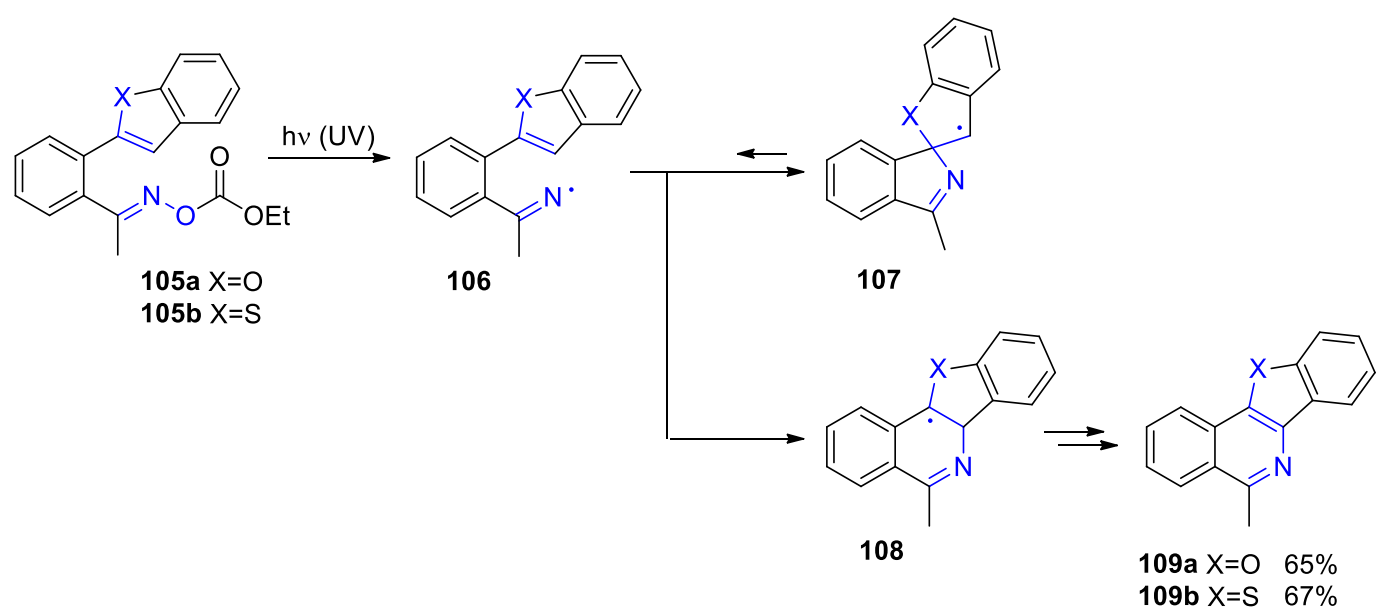

Scheme 20. Synthesis of phenanthridines with benzofuran and benzothiophene substituted oxime derivatives.

Addition cyclization reactions were carried out. In such an example, a nitrogen centered radical intermediate $\mathbf{1 1 1}$ is generated from a corresponding oxime $\mathbf{1 1 0}$ (Scheme 21). ${ }^{54}$ After addition of an alkyne 114, a vinyl radical $\mathbf{1 1 2}$ is formed. The final isoquinoline derivative $\mathbf{1 1 3}$ is then obtained in a cyclization step.

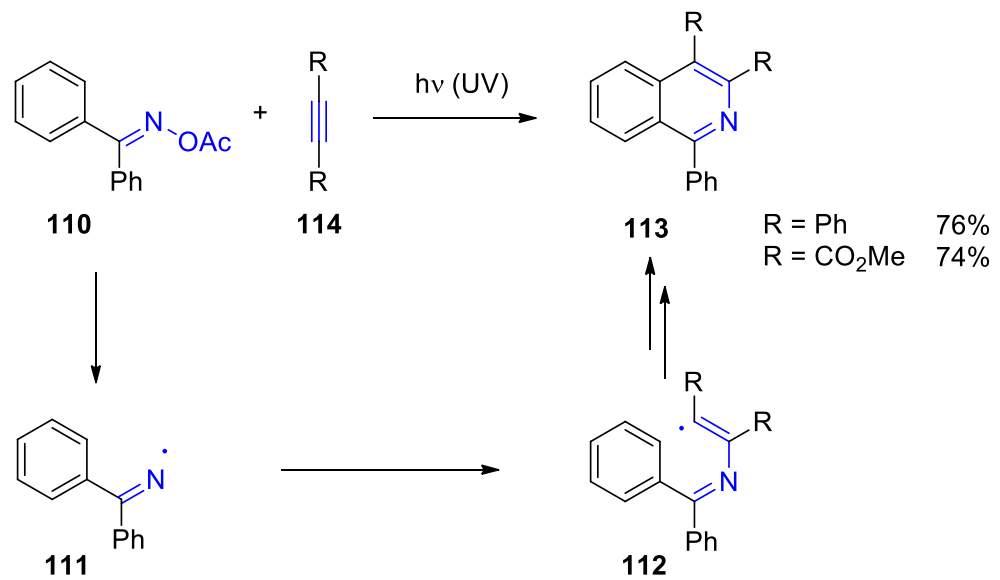

Scheme 21. Radical addition cyclization applied to the synthesis of isoquinoline derivatives. 


\subsection{Generation of radical intermediates by irradiation of charge transfer complexes}

Excitation of a ground state charge transfer complex is now applied to the formation of radical intermediates. Such a reaction was carried out with visible light irradiation (Scheme 22). ${ }^{55}$ In this case, the oxime function in $\mathbf{1 1 5}$ carries a dinitrophenyl substituent as leaving group. The intramolecular radical addition takes place on a phenol substituent. The resulting spiropyrroline derivatives $\mathbf{1 1 6}$ were obtained in yields up to $93 \%$. In the present paper 27 examples have been published. It is a typical example of a dearomtization reaction. Such transformations are often used to increase structural complexity. Visible light irradiation is also recommended since the dienone moiety in $\mathbf{1 1 6}$ undergoes photochemical rearrangements under UV irradiation. Furthermore, the reaction has been carried out in the presence of DBU. It has been shown that DBU deprotonates the phenol moiety and generate a charge transfer complex with dinitrophenyl substituent on the oxime function (117). Both the phenolate and the charge transfer complex significantly increase the light absorption in the visible domain (450-600 nm). After photochemical excitation, electron transfer takes place in the charge transfer moiety leading to a radical ion pair 118. ${ }^{56,57}$ The cleavage of the $\mathrm{N}-\mathrm{O}$ bond of the oxime and the release of dinitrophenolate 119 yields the nitrogen centered radical intermediate 120. The latter undergoes cyclization (121) and after transfer of an electron to the radical cation of DBU, the final product $\mathbf{1 1 6}$ is formed.
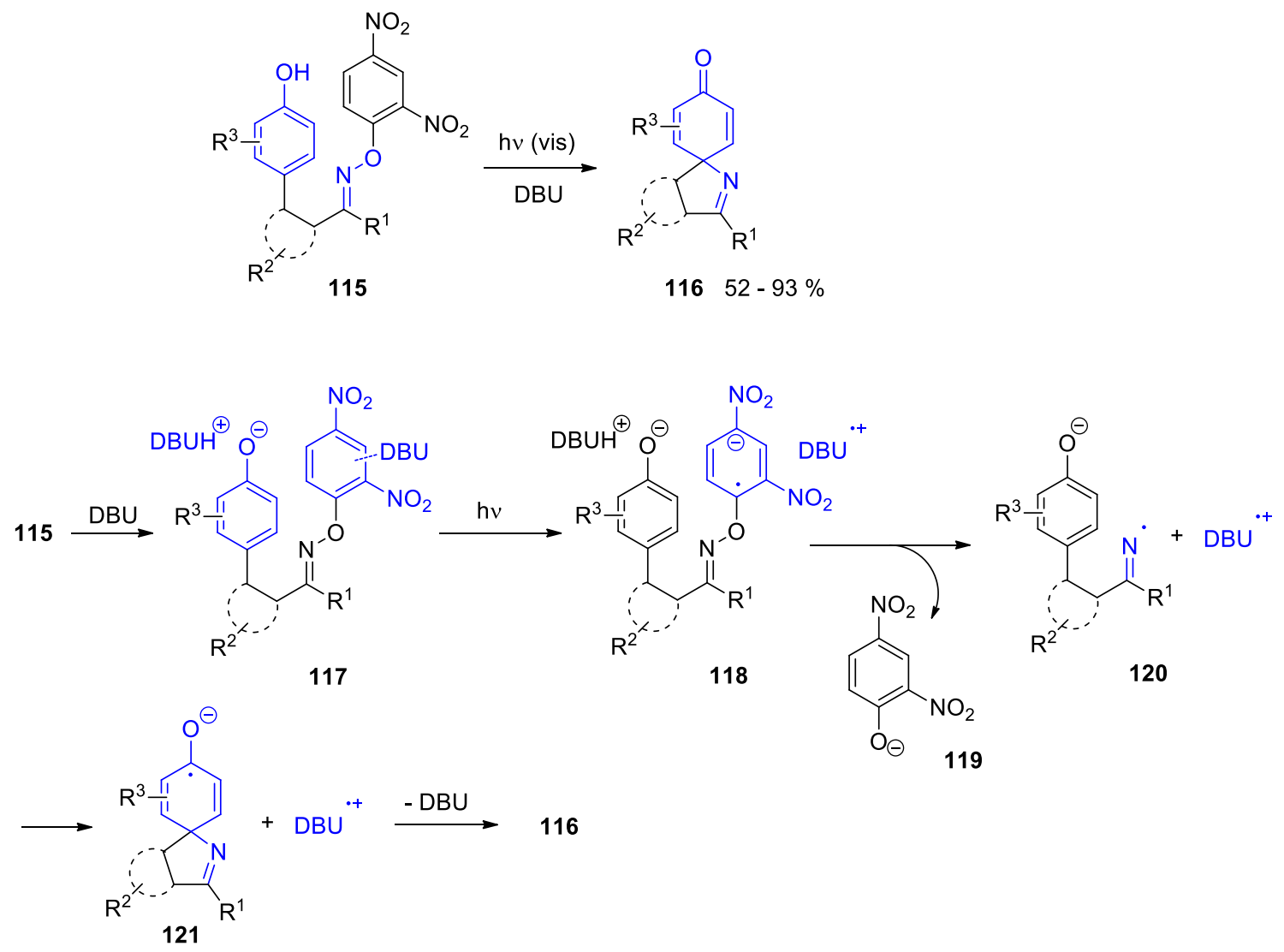

Scheme 22. Synthesis of spiropyrrolines. The cleavage of the $\mathrm{N}-\mathrm{O}$ bond is induced by visible light absorption of a charge transfer complex 117. 
A similar reaction with an intramolecular charge transfer interaction between tertiary amines and the 2,4-dinitrophenyl protecting group has also been reported. A typical example is presented in Scheme $23 .{ }^{58}$ After light absorption of the charge transfer complex 122, an intramolecular electron transfer takes place leading to the intramolecular radical ion pair 123. N-O Bond fragmentation occurs and 2,4dinitrophenolate 119 is released. In the resulting iminyl radical intermediate 124, an intramolecular hydrogen transfer leads to imine iminium species 125 . Nucleophilic addition and deprotonation yield the dihydroimidazole 126. Under the reaction conditions, oxidation takes place and the imidazole $\mathbf{1 2 7}$ is isolated in good yield. This convenient imidazole synthesis was carried out with 27 substrates leading to the corresponding imidazole derivatives with yields up to $85 \%$. If a corresponding pyrrolidine substrate was used instead of a piperidine, no oxidation in the final step was observed. The resulting dihydroimidazoles were isolated in good yields.<smiles>COc1ccc(C(=N)C[N+]2CCCCC2)cc1</smiles>

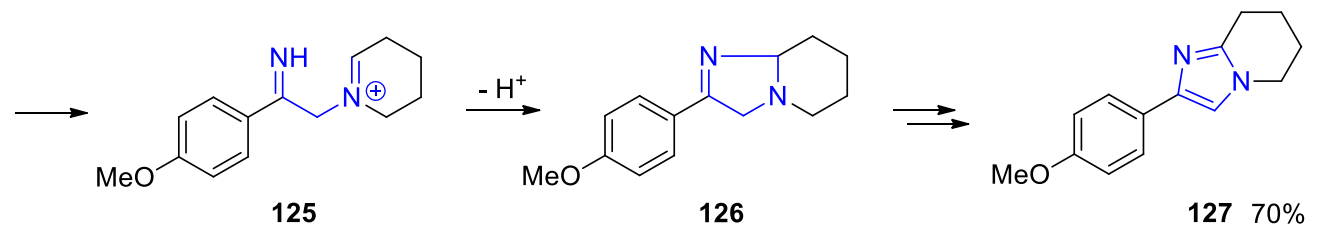

Scheme 23. Synthesis of imidazoles using excitation of an intramolecular charge transfer complex of a tertiary amine and a dinitrophenyl moiety.

Using the above-mentioned activation by light absorption of an intermolecular charge transfer complex with a dinitrophenyl substituted oxime function (128), the radical intermediate $\mathbf{1 2 9}$ is generated by the release of dinitrophenolate (Scheme 24$).{ }^{59}$ Cyclization yields the intermediate $\mathbf{1 3 0}$. This intermediate is trapped by $\mathrm{SO}_{2}$ and various silyl enol ethers 131 leading to the final product 132. A variety of aromatic substrates have been successfully transformed. Also, heteroaromatic compounds ( $\mathrm{Ar}^{1}=2$-furyl) can be obtained. In the present report, 20 different derivatives have been transformed with yields up to $92 \%$. 


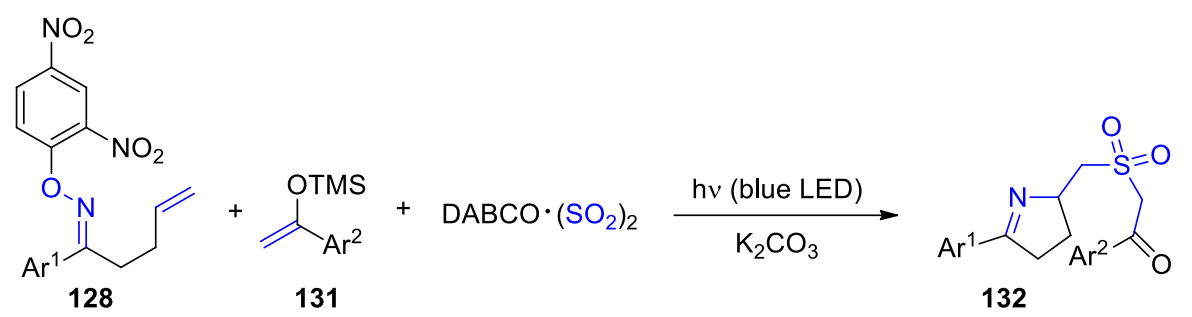<smiles>C=CCCC(=[W])C(C)C(C)C</smiles>

Scheme 24. Synthesis of pyrrolines using radical trapping with $\mathrm{SO}_{2}$ and silyl enol ethers.

\subsection{Generation of radical intermediates by photoredox catalysis.}

Using photoredox catalysis with an iridium complex, a one pot procedure for the synthesis of phenanthridines has been realized (Scheme 25). ${ }^{60}$ In the first acid catalyzed step, the oxime derivative $\mathbf{1 3 5}$ is formed by condensation of $\mathbf{1 3 3}$ and 134. In the photochemical step, the nitrogen centered radical 136 is generated. Cyclization leads to the final product 137. Under these mild reaction conditions, also benzonaphtyridines such as $\mathbf{1 3 8}, \mathbf{1 3 9}$ or $\mathbf{1 4 0}$ have been synthesized. Starting from corresponding precursors, a variety of quinoline derivatives such as $\mathbf{1 4 1}$ and $\mathbf{1 4 2}$ are accessible. In the present report, the preparation of 28 derivatives with yields up to $89 \%$ has been described. The reaction sequence was also applied to the synthesis of benzophenanthridine alkaloids and pyridines. ${ }^{61}$

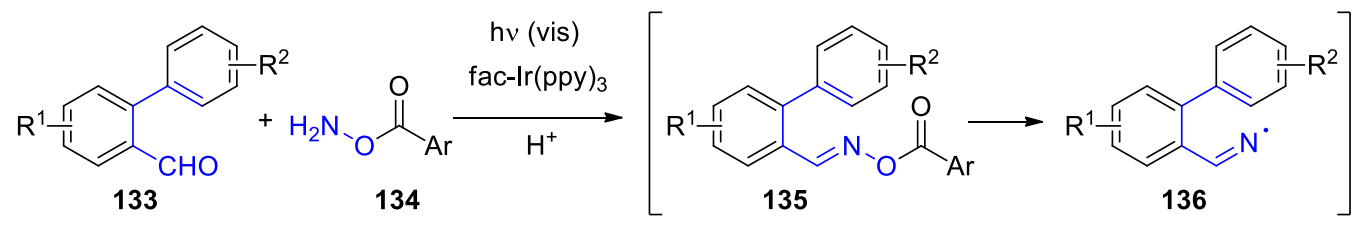<smiles>[R][R1]1ccc2cnc3c(c2c1)=CC=CC([R])=C3</smiles><smiles></smiles><smiles>FC(F)(F)c1ccc2c3c(ncc2c1)CCCC3</smiles><smiles>Fc1ccc(-c2cncc3cc(F)ccc23)cc1</smiles>

Scheme 25. Photoredox catalytic synthesis of phenanthridines and quinoline derivatives. 
The oxime function can also be protected with phenyl substituents. In this case, the cleavage of the $\mathrm{N}-\mathrm{O}$ bond is accompanied with the release of phenol or phenolate. In the photochemical transformation of 143 in the presence of 1,5-dimethoxynaphthalene (DMN), a photochemical electron transfer from this donor molecule to $\mathbf{1 4 3}$ is involved (Scheme 26). ${ }^{62}$ The nitrogen centered radical $\mathbf{1 4 4}$ is thus generated. Cyclization leads to the bicyclic radical 145. After hydrogen abstraction from 1,4-cyclohexadiene, the final products 146 are obtained. Recently, similar reactions have been carried out using photoredox catalysis with visible light. ${ }^{56}$ In this case also simple ketones such as chloroanthraquinone can be used as sensitizer or photocatalyst. ${ }^{63}$ Detailed theoretical and physicochemical studies of such reactions have been carried out. ${ }^{64}$

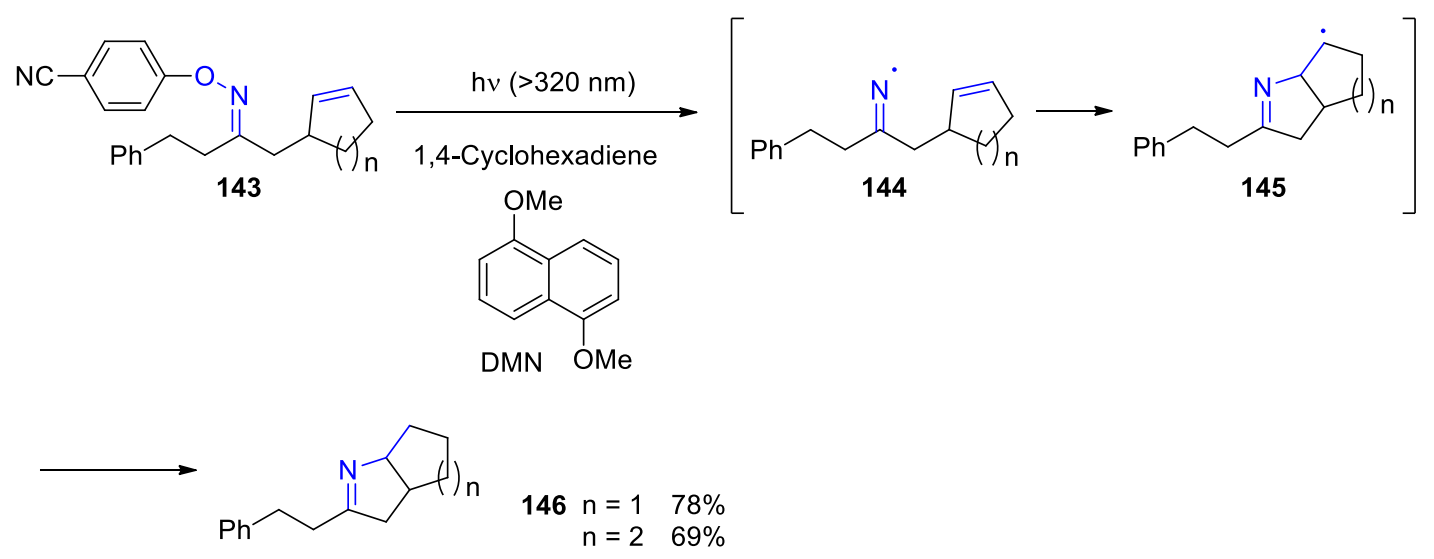

Scheme 26. Photoredox catalyzed synthesis of hexahydrocyclopenta[b]pyrroles and hexahydroindoles.

Radical species which result from a cyclization step can be trapped by various reagents. This leads to a further increase of molecular complexity and diversity. A large variety of pyrrolines have been synthesized starting from 147 in which the oxime group is O-alklylated by lactic acid (Scheme 27). ${ }^{65}$ During the reaction, pyrroline radical intermediates 148 are formed. Several electron deficient alkenes 149 are added which leads to the final products $\mathbf{1 5 0}$. More than 30 compounds $\mathbf{1 5 0}$ have been reported in the present investigation. The products were isolated with yields up to $91 \%$. Various carboxylic acid derivatives haven been used as leaving groups such as oxalic acid ${ }^{66}$ or groups enabling Barton decarboxylation. ${ }^{67}$ 
149

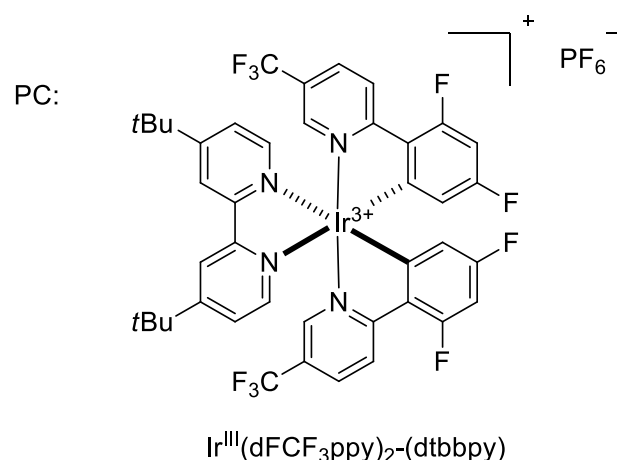

Scheme 27. Synthesis of a large variety of pyrrolines starting form oximes. Photoredox catalysis is involved.

The following photoredox catalysis mechanism has been suggested (Scheme 28). After photochemical excitation of the $\operatorname{Ir}($ III) complex, the carboxylate function in $\mathbf{1 5 1}$ is oxidized. An $\operatorname{Ir}(\mathrm{II})$ species is formed. The resulting neutral radical $\mathbf{1 5 2}$ immediately undergoes fragmentation; carbon dioxide and acetaldehyde are released. Cyclization of the resulting iminyl radical $\mathbf{1 5 3}$ yields the alkyl radical $\mathbf{1 5 4 .}$ Addition of the electron deficient alkene leads to the electrophilic radical $\mathbf{1 5 5}$. The latter is reduced by the $\operatorname{Ir}(I I)$ species. In this step, the photoredox catalyst is regenerated. Protonation of the anion intermediate 156 yields the final product 157. 


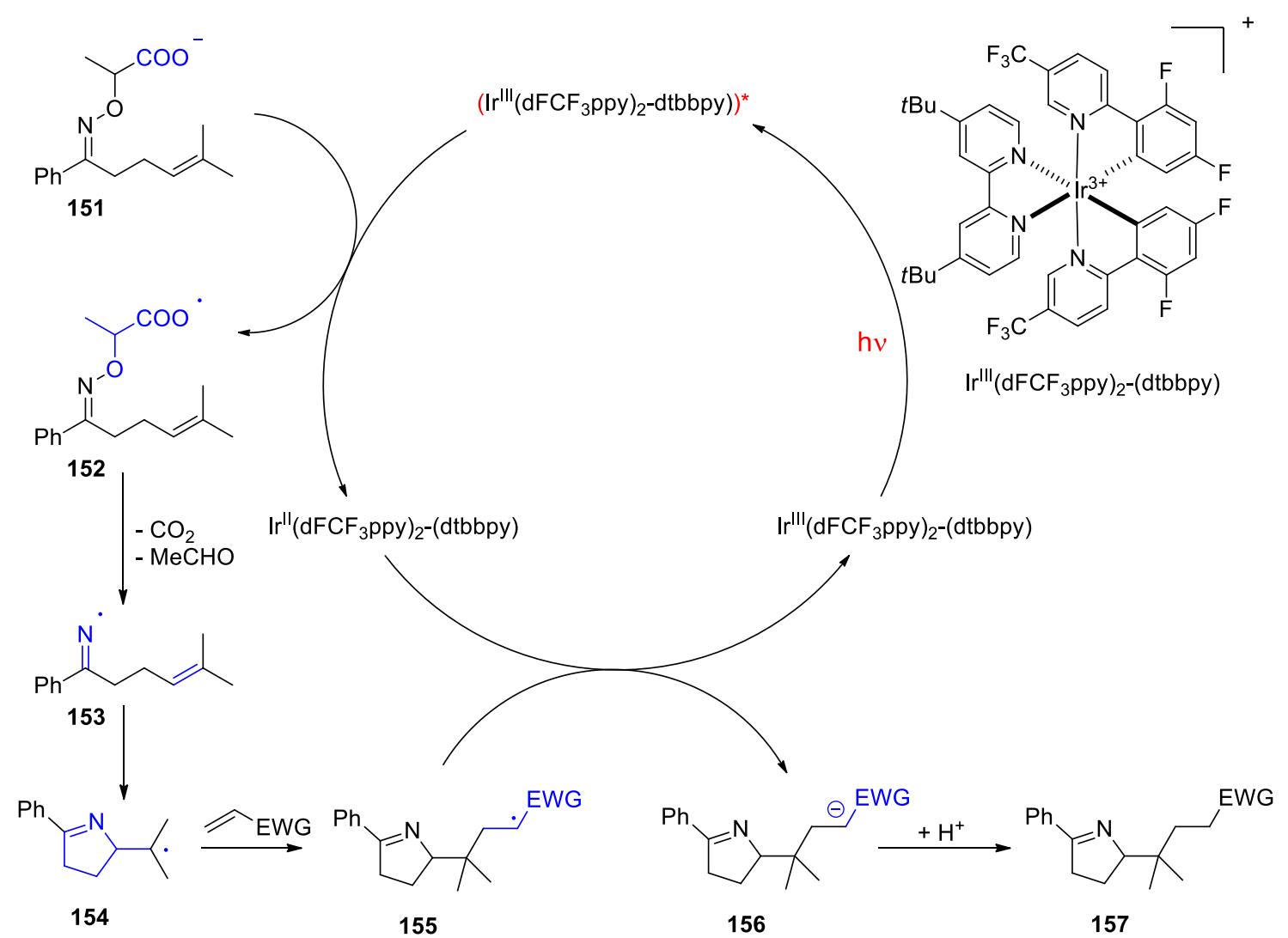

Scheme 28. Proposed mechanism for formation of pyrrolines in the photo-redoxcatalytic transformation of oximes with electron-deficient alkenes.

Very similar reactions have been carried out with silyenolethers as trapping reagent. In such an investigation, also a large variety of pyrrolines have been synthesized. ${ }^{68}$ About 35 examples are reported. The products are obtained with yields up to $83 \%$. Also, styryl borates have been successfully used as trapping reagent. ${ }^{69}$

Various trapping reagents enables the introduction of a large number of functional groups. A selection of pyrroline derivatives from one particular investigation is depicted in Figure $1 .{ }^{70}$ The reactions have been carried out using photo redox catalysis with $\mathrm{N}$-methyl mesitylacridinium perchlorate. The usefulness of this synthetic approach is also demonstrated in the modification of natural products (Scheme 29). ${ }^{71}$ The morphine derivative $\mathbf{1 5 8}$ was readily transformed into the corresponding dihydropyrroline compounds 160,161 and 162 . The nitrogen centered radical 159 is the key intermediate in these reactions. 


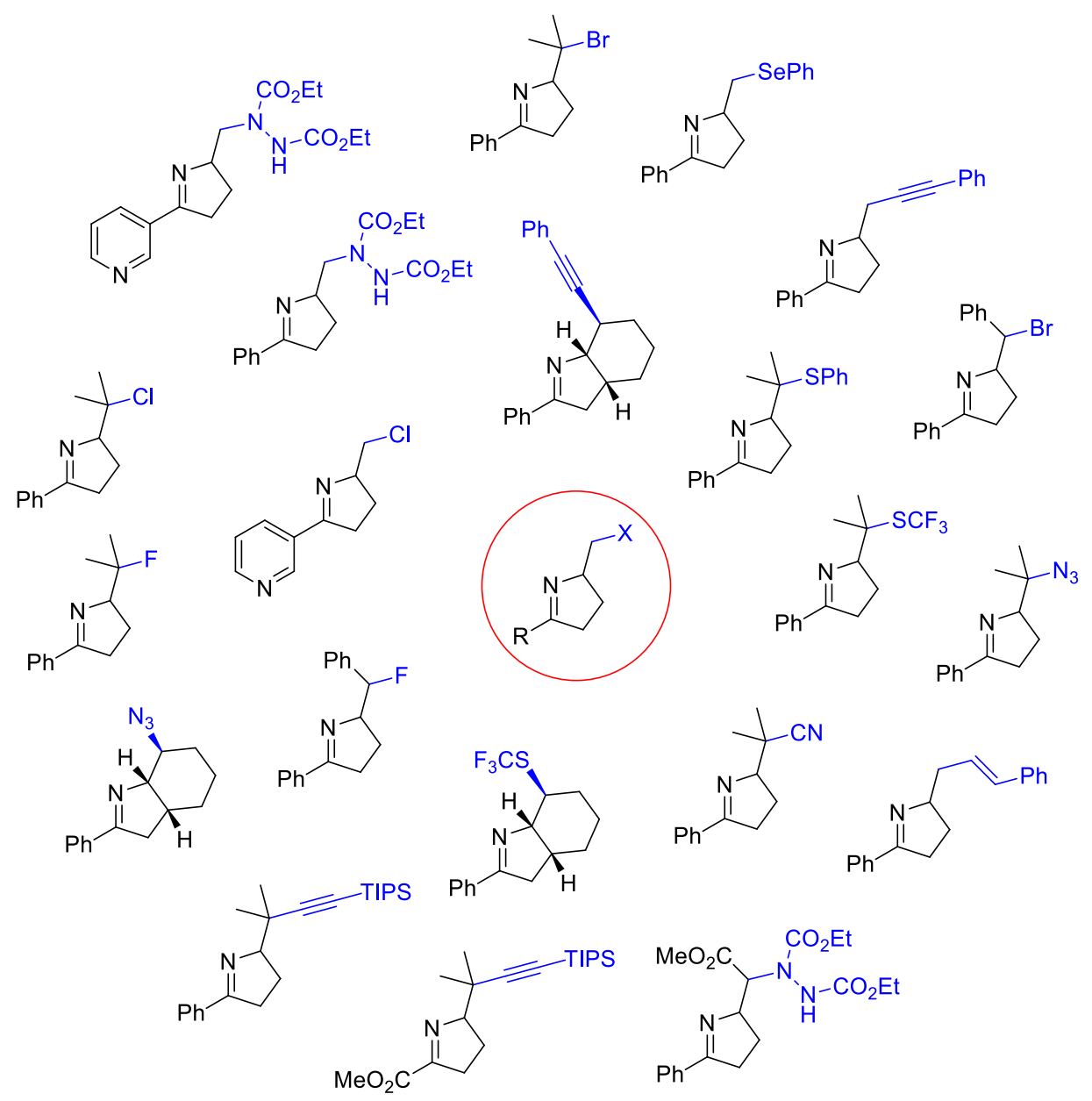

Figure 1. Structural diversity in a pyrroline family is generated by variation of the trapping reagent.
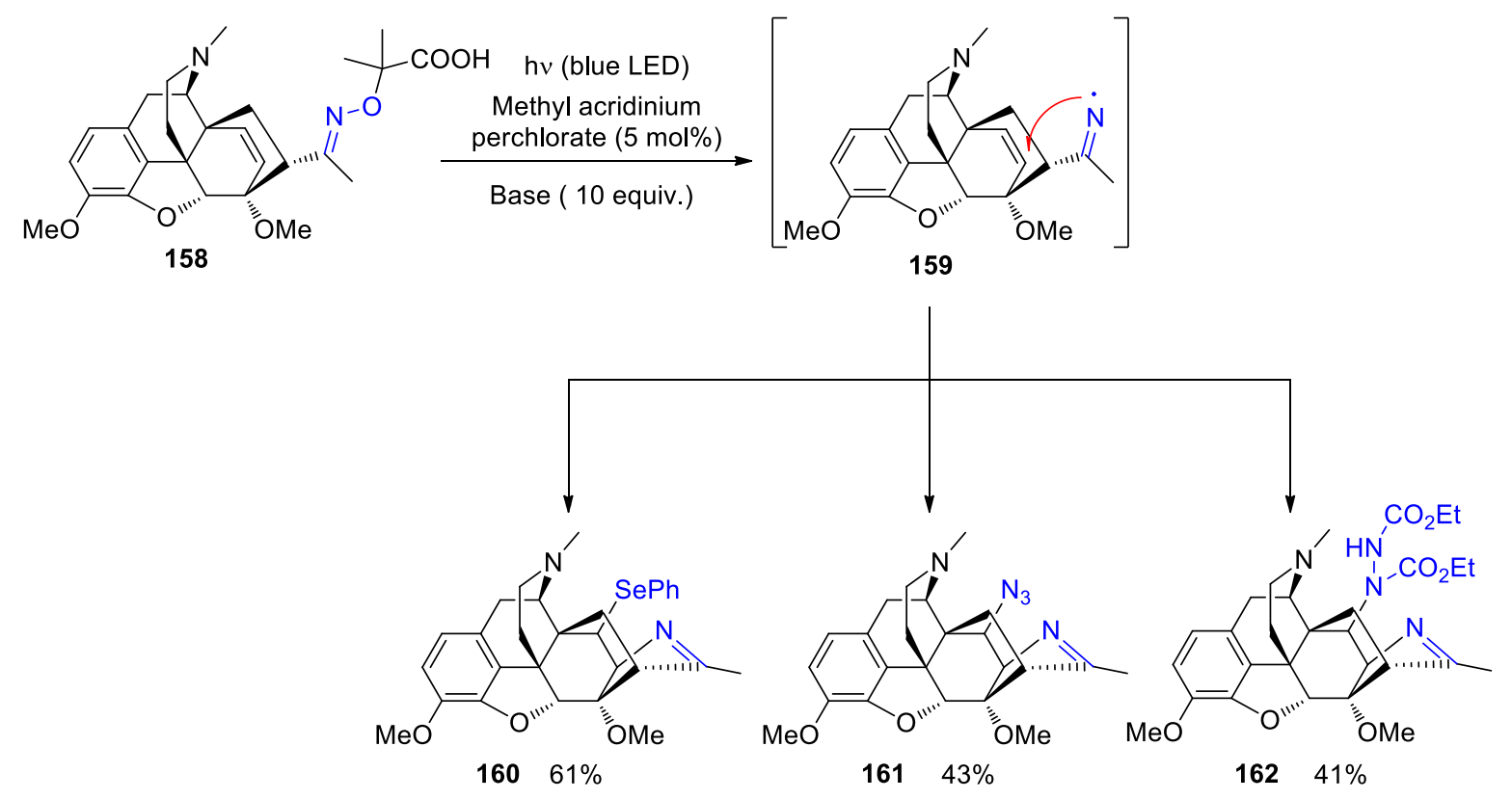

Scheme 29. Transformation of morphine derivative into corresponding dihydropyrrole compounds. 
Photoredox catalysis is often combined with organometallic catalysis. ${ }^{71}$ In such a reaction, oximes like 163 have been transformed into dihydropyrroles 164 (Scheme 30 ). ${ }^{72}$ As previously discussed, a dihydropyrrole radical intermediate $\mathbf{1 6 5}$ is generated in a photoredox catalytic process. It is trapped by a cobalt complex (166) and elimination of a cobalt species $([\mathrm{Co}] \mathrm{H})$ leads to the final product $\mathbf{1 6 4}$. Under similar conditions and in the presence of alkenes the latter are added leading to corresponding radicals 167 which are then trapped by a cobalt complex (168). Elimination of a cobalt species leads to the final products 169. Around 60 examples are reported in the present study. The products have been isolated in yields up to $95 \%$ for simple cyclizations and yields up to $77 \%$ for cyclization with subsequent addition to alkenes.
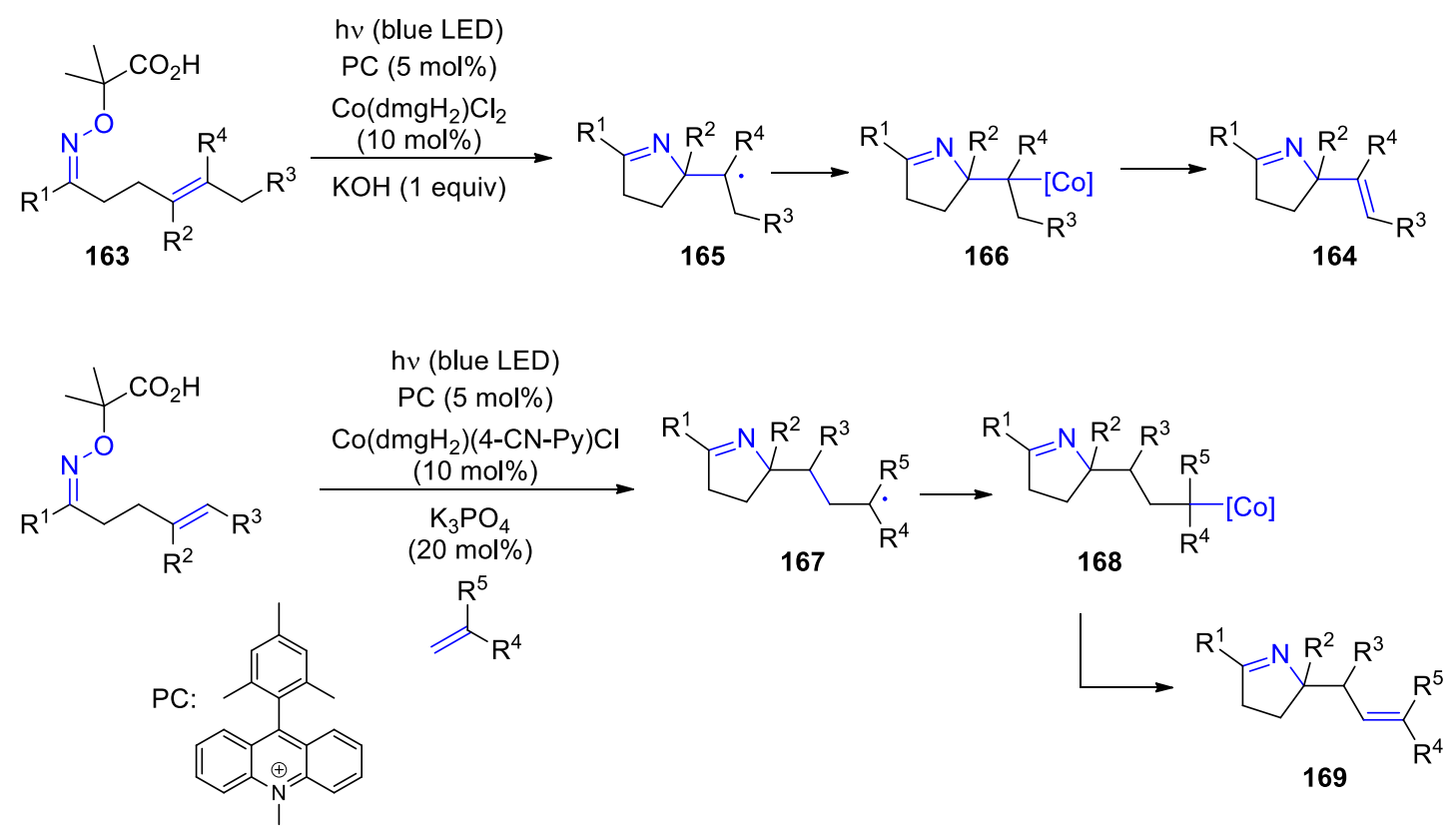

Scheme 30. Photoredox-organometallic dual catalysis applied to the synthesis of dihydropyrrols from oxime derivatives.

Radical addition may also occur at the carbon atom of the oxime function. In a photoredox catalytic reaction, styrene derivatives $\mathbf{1 7 0}$ have been added to oximes from $\alpha$-keto or glyoxylic acid $\mathbf{1 7 1}$ yielding butyrolacton compounds 172 (Scheme 31). ${ }^{73}$ In the present report 21 different butyrolactones have been synthesized with yields up to $88 \%$. The products can easily be reduced to the corresponding $\alpha$-amino lactones. The reaction starts with the excitation of the acridinium photo catalyst PC. At the excited state, it is capable of oxidizing the alkene $\mathbf{1 7 0}$ and radical cation $\mathbf{1 7 3}$ is generated. The latter reacts with the glyoxylic acid oxime $\mathbf{1 7 1}$ leading to the intermediate $\mathbf{1 7 4}$. Deprotonation by an amine base and radical cyclization yields intermediate 175. Hydrogen from thiophenolate is transferred and the final product $\mathbf{1 7 2}$ is obtained. The concomitantly generated phenylthiyl radical $\mathbf{1 7 6}$ oxidizes the reduced species 177 of 
the acridinium photocatalyst which is thus regenerated. Phenylthiyl radicals $\mathbf{1 7 6}$ are also generated by photochemical fragmentation of diphenyl disulfide. Thiyl radicals play a key role as mediators in many radical reactions. ${ }^{74}$
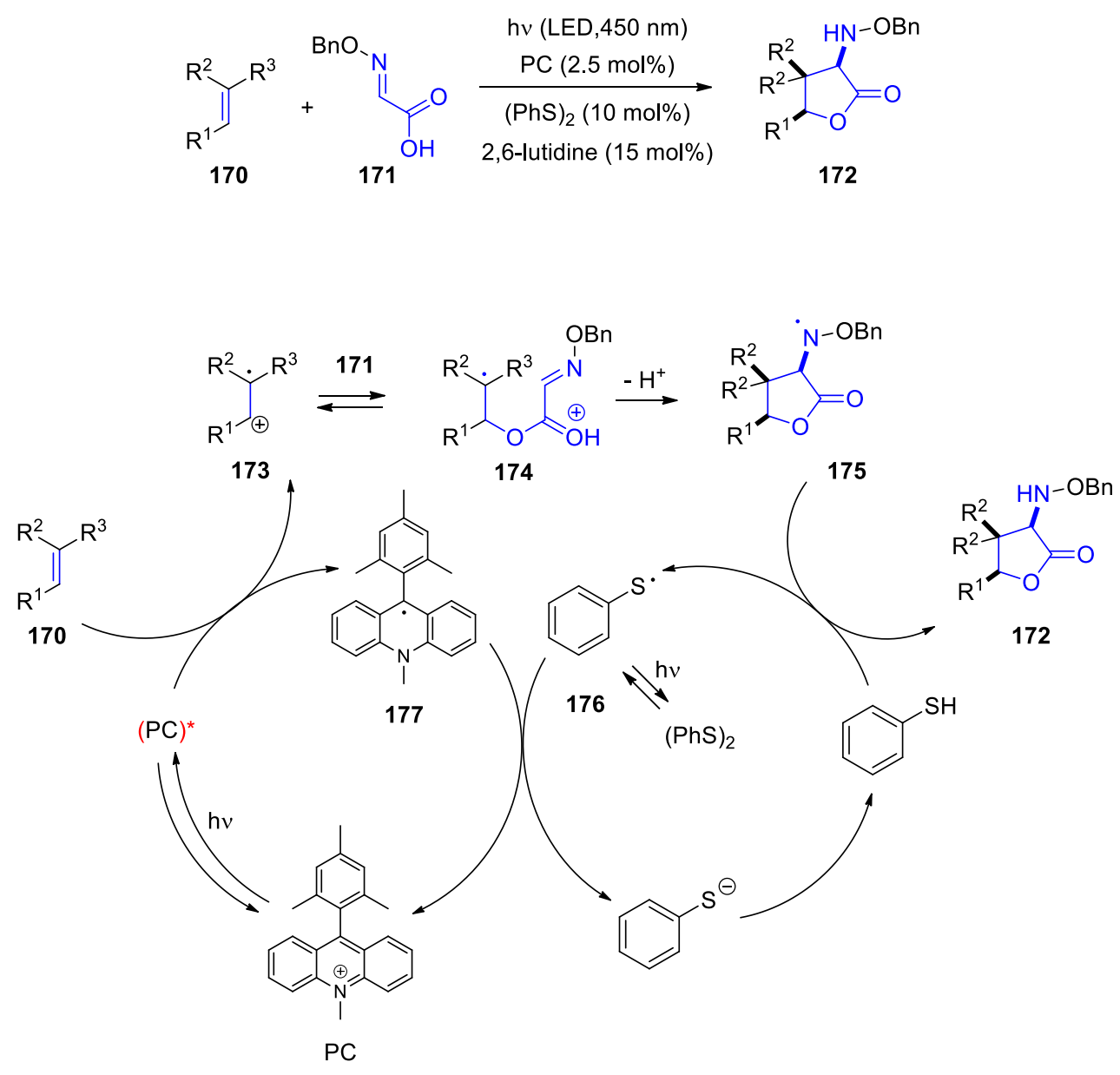

Scheme 31. Photo redox catalytic synthesis of butyrolactones form alkenes and glyoxylic acid oximes.

\section{Chloroimino derivatives}

\subsection{Generation of radical intermediates by electronic excitation of the substrates}

The acyl substituents of the oxime function can be replaced by other leaving groups such as halogens. For example, chloroimino derivatives like $\mathbf{1 7 8}$ undergo photochemically induced radical cyclization leading to quinoxalinones 179 (Scheme 32). ${ }^{75}$ The reaction was carried out with visible light in the presence or the absence of a ruthenium base photoredox catalyst. Depending on the substitution pattern either the first or the second variant was more efficient. The reaction has been carried out with 20 different substrates. The quinoxalinones $\mathbf{1 7 9}$ have been isolated with yields up to $97 \%$. As previously explained, the iminyl radicals $\mathbf{1 8 0}$ are generated. Radical cyclization may occur either in the ipso position 
(181) or the ortho position (182) of the aromatic ring. The first reaction mode is kinetically favored but reversible. Furthermore, a rearrangement of $\mathbf{1 8 1}$ into $\mathbf{1 8 2}$ was discussed. Thus generally, the final products 179 result from ortho cyclization.
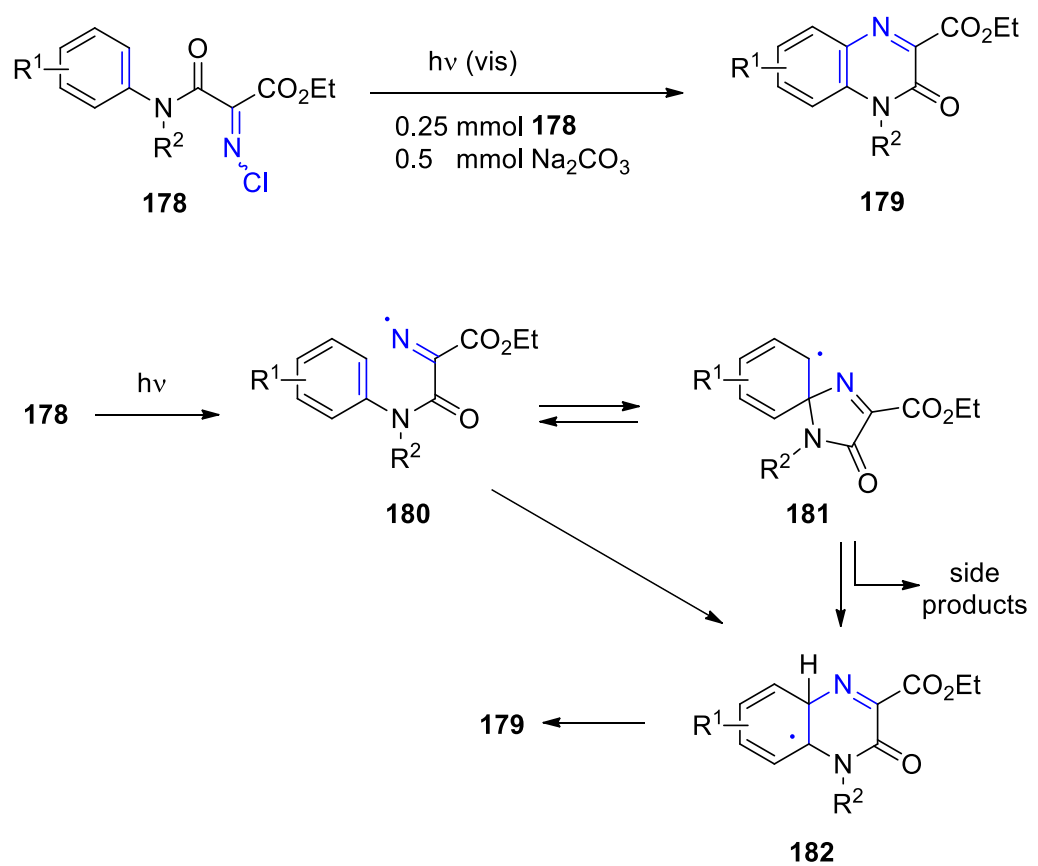

Scheme 32. Synthesis of quinoxalinones using radical cyclization with chloroimino derivatives.

\subsection{Generation of radical intermediates by photoredox catalysis.}

An interesting observation was made for the transformation of compound $\mathbf{1 8 3}$ (Scheme 33). ${ }^{76}$ When the reaction was carried out in the presence of the photoredox catalyst $\mathrm{Ru}\left(\right.$ phen) ${ }_{3} \mathrm{Cl}_{2}$, mainly compounds 184 and $\mathbf{1 8 5}$ resulting from spiro cyclization at the p-methoxyphenyl substituent were isolated. Addition of the electrophilic nitrogen centered radical $\mathbf{1 8 6}$ with the electron richer aryl substituent is indeed favored since polar effects have an influence on radical reactions. ${ }^{2}$ Under photoredox catalytic conditions, the resulting radical intermediate 187 is oxidized and the cation intermediate 188 leads to 184 and $\mathbf{1 8 5}$. Radical cyclization at the phenyl ring leads to minor amounts of the quinoxalinone 189. Only traces of the corresponding quinoxalinone $\mathbf{1 9 0}$ resulting from ortho cyclization at the p-methoxyphenyl substituent were obtained. It should be mentioned that in the absence of a photoredox catalyst, the spirocyclic intermediate 187 cannot be oxidized. In this case, only the quinoxalinones 189 and 190 were formed in comparable amounts owing to the reversible formation of the intermediate $\mathbf{1 8 7}$ or $\mathbf{1 8 1}$ (Scheme 32). 
<smiles>CCOC(=O)C(=N)C(=O)N(c1ccc(OC)cc1)c1ccc(C(=O)OCC)cc1</smiles><smiles>CCOC(=O)c1nc2ccccc2n(-c2ccc(OC)cc2)c1=O</smiles><smiles>CCOC(=O)C1=NC2(C=CC(OC)C=C2)N(c2ccccc2)C1=O</smiles>

Scheme 33. Competitive formation of sprio cyclic products $\mathbf{1 8 4}$ and $\mathbf{1 8 5}$ in the radical cyclization with chloroimino derivatives.

\section{Hydrazones}

In the case of hydrazone derivatives, radical reactions can be carried out at the carbon atom or at the nitrogen atoms, which provide numerous perspectives for applications to the synthesis of heterocycles. ${ }^{76}$ A remarkable radical cyclization has been reported for the keto hydrazone 191 (Scheme 34). ${ }^{77}$ The reaction is carried out in the presence of the chiral phosphoric acid derivative 193 involving photoredox catalysis with the iridium complex 194. The Hantzsch ester 195 is used as hydrogen donor. The hydroxyl hydrazine product 192 was obtained in high yield and almost complete enantioselectivity. 


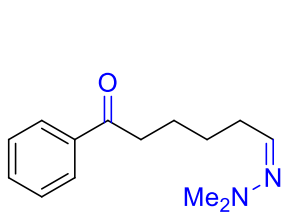

191

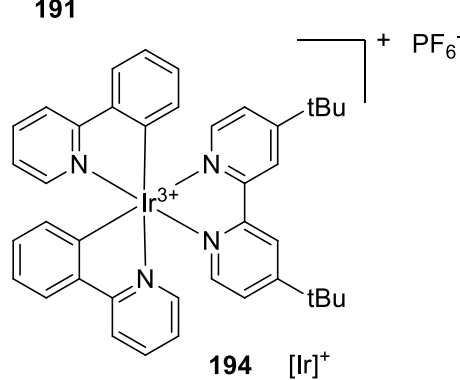

hv (vis)

$194(5 \mathrm{~mol} \%)$

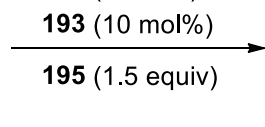

$\mathrm{PF}_{6}^{-}$

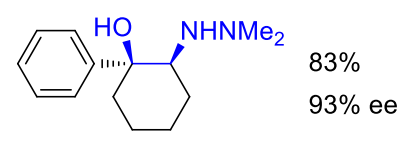

192

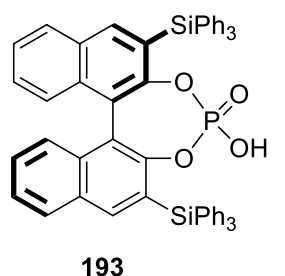

193<smiles></smiles>

195

Scheme 34. Enantioselective photopinacolization like reaction of a ketohydrazone.

The following mechanism has been discussed. After Photochemical excitation of the photo-catalyst, electron transfer occurs from the Hantzsch ester derivative to the catalyst leading to the $[\mathrm{Ir}]^{0}$ species (Scheme 35). This step initiates the catalytic cycle. Electron transfer from the $[\mathrm{Ir}]^{0}$ species to the ketone function leads to the intermediate 196. It must be pointed out that this transfer is coupled to a proton transfer from the phosphoric acid derivative 194. Aside many chemical processes, such proton coupled electron transfer (PCET) processes are now widely reported in photoredox catalytic reactions. ${ }^{32}$ In the present case, this step is exergonic by $5.2 \mathrm{kcal} / \mathrm{mol}$ as it was determined by calculations. C-C bond formation leads to the intermediate 197 with a tertiary alcohol function and a nitrogen centered radical. During this step, the phosphoric acid derivative 193 remain complexed to the intermediates in the way that efficient chiral induction occurs. The final product $\mathbf{1 9 2}$ is formed by hydrogen atom transfer from the Hantzsch ester 195. In an additional photochemical process, involving electron and proton transfer steps, the pyridine derivative $\mathbf{2 0 0}$ is formed via intermediates $\mathbf{1 9 8}$ and $\mathbf{1 9 9}$ and the catalytically active species $[\mathrm{Ir}]^{0}$ is generated. 


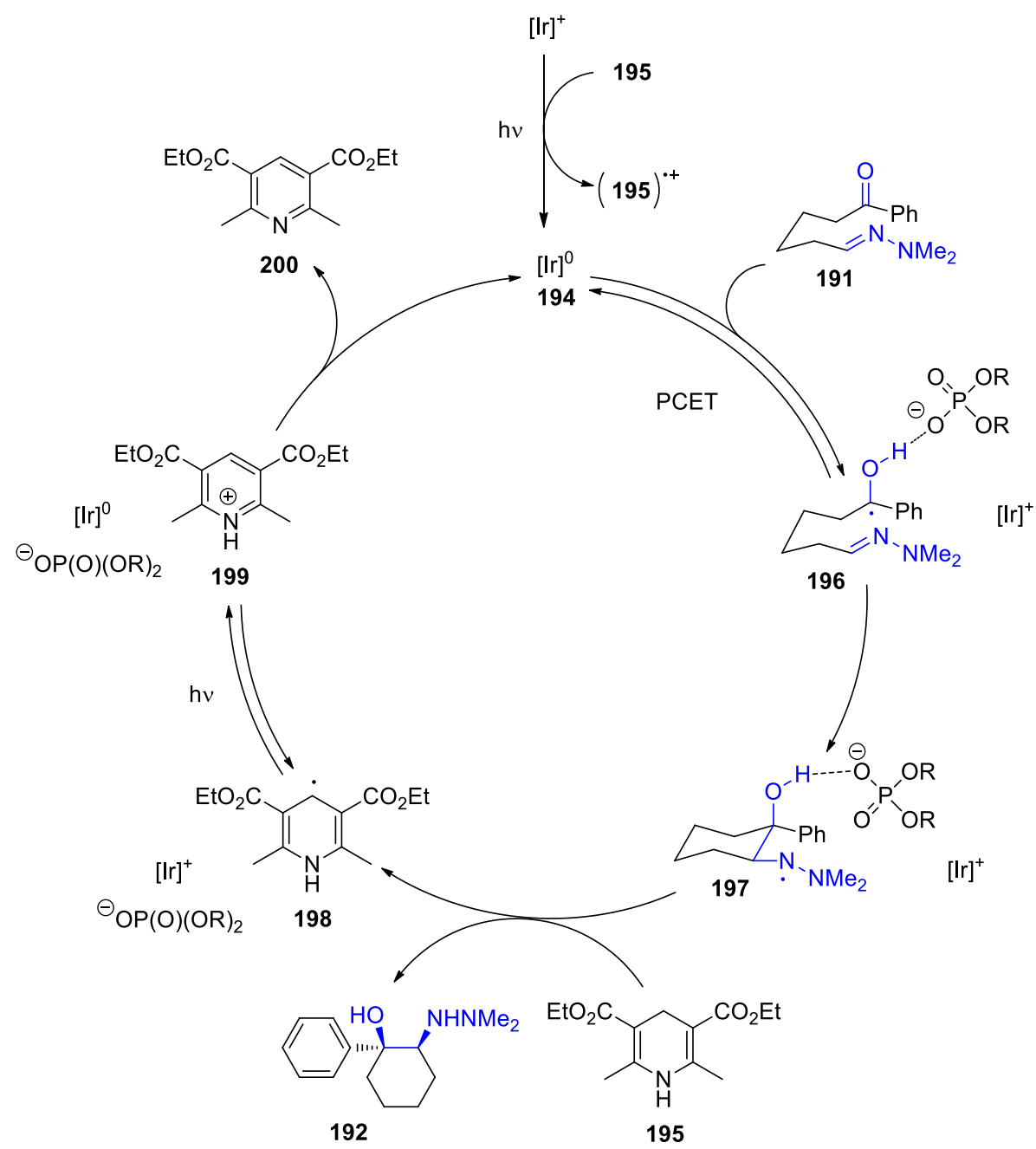

Scheme 35. Suggested mechanism of the enantioselective photo-redox catalytic mixed pinacolization analogue cyclization (Scheme 34).

Consecutive radical addition cyclization reactions lead to complex polycyclic compounds. In a photoredox catalytic reaction, the hydrazone derivative 201 carrying an alkyne substituent was transformed with the malonic ester derivative 202 (Scheme 36). ${ }^{78}$ Two rings are formed in this reaction (203). The products were obtained in yields up to $92 \%$. The synthesis of 20 different products by this method has been reported. The reaction has been carried out on gram scale. It can also be used for the preparation of polycyclic pyridine derivatives. The reaction starts with the excitation of the iridium photocatalyst which is now capable of reducing the malonic ester bromide 202. The radical intermediate $\mathbf{2 0 4}$ is generated by release of bromide. Addition of this radical to the alkyne function (205) and double cyclization yields the nitrogen centered radical 206. Oxidation of the latter leads to the corresponding cation 207. After deprotonation, the final product 203 is obtained. 

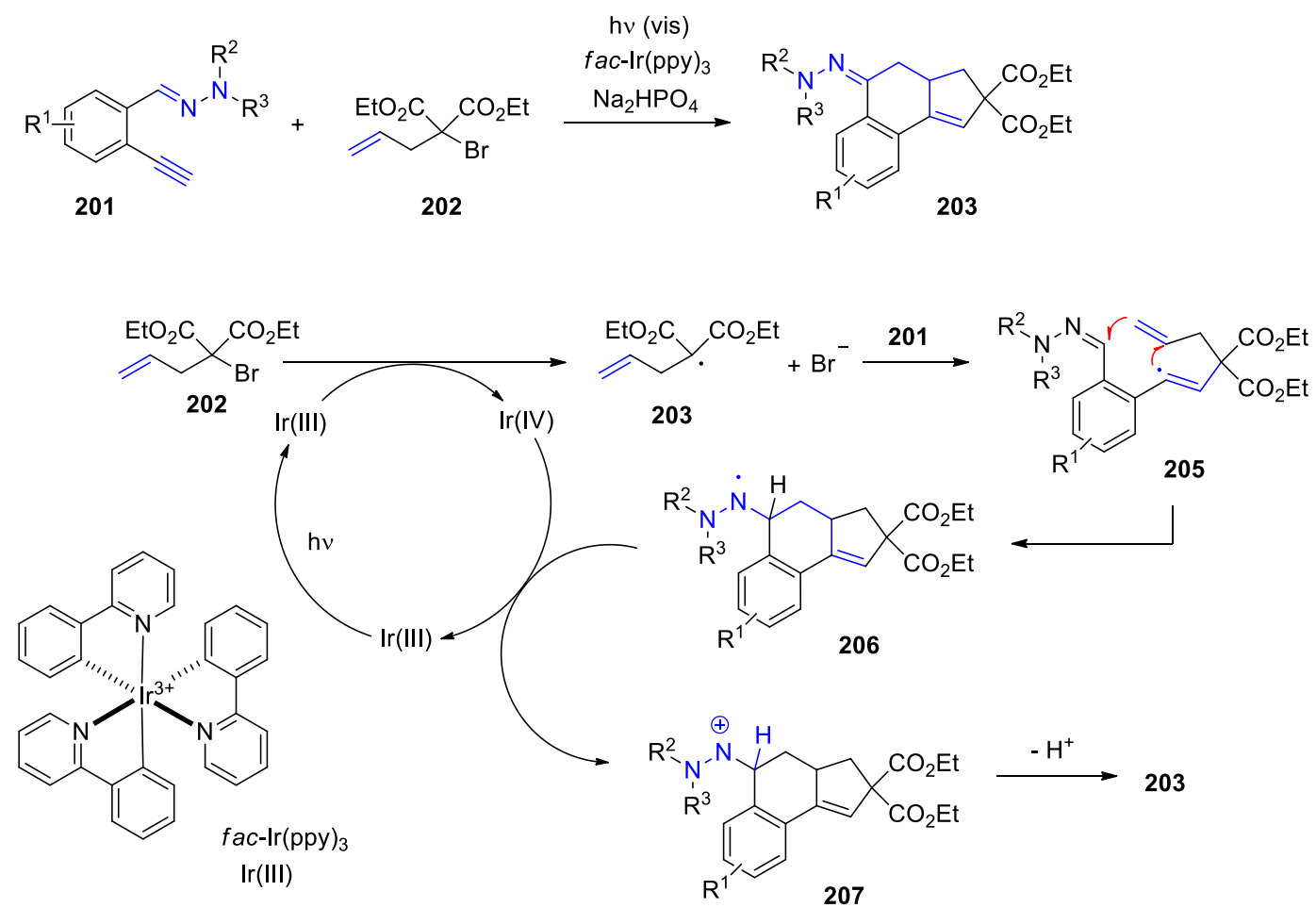

Scheme 36. Radical addition cyclization reaction leading to cyclohexylidenehydrazine-fused polycycles.

Cyclization reactions are also observed at the amine nitrogen of a hydrazone function. ${ }^{79}$ Hydrazone derivatives such as $\mathbf{2 0 8 a , b}$ or $\mathbf{2 1 0}$ (Scheme $\mathbf{3 7}$ ) undergo radical cyclization leading to dihydropyrazoles such as $\mathbf{2 0 9 a , b}$ or to dihydropyridazine such as $\mathbf{2 1 1}$ or tetrahydropyrazines $\mathbf{2 1 2}{ }^{80}$ Photoredox catalysis with $\mathrm{Ru}(\mathrm{bpy})_{3} \mathrm{Cl}_{2}$ was applied to generate the nitrogen centered radicals $\mathbf{2 1 4}$ from the 213. In the case of 208a,b, only exocyclization was observed leading to the radical intermediates $\mathbf{2 1 5}$ and the final products $\mathbf{2 0 9 a , b}$ were isolated. In the case of $\mathbf{2 1 0}$, both exo and endo cyclization were observed. Thus products 211, $\mathbf{2 1 2}$ and $\mathbf{2 1 6}$ are formed. It should be pointed out that $\mathbf{2 1 6}$ is formed by trapping of the primary radical 215 by a dichloromethyl radical. Calculations revealed that exo cyclization is kinetically favored and thermodynamically disfavored in the case of $\mathbf{2 0 8 a , b}$. The reaction conditions have further been optimized. In the presence of TEMPO, radical intermediates $\mathbf{2 1 5}$ are trapped. Elimination of TEMPO-H then yields dihydropyrazoles. ${ }^{81}$ Various trapping reagents favors the formation of corresponding dihydropyrazoles or tetrahydropyrazoles. ${ }^{82}$ 

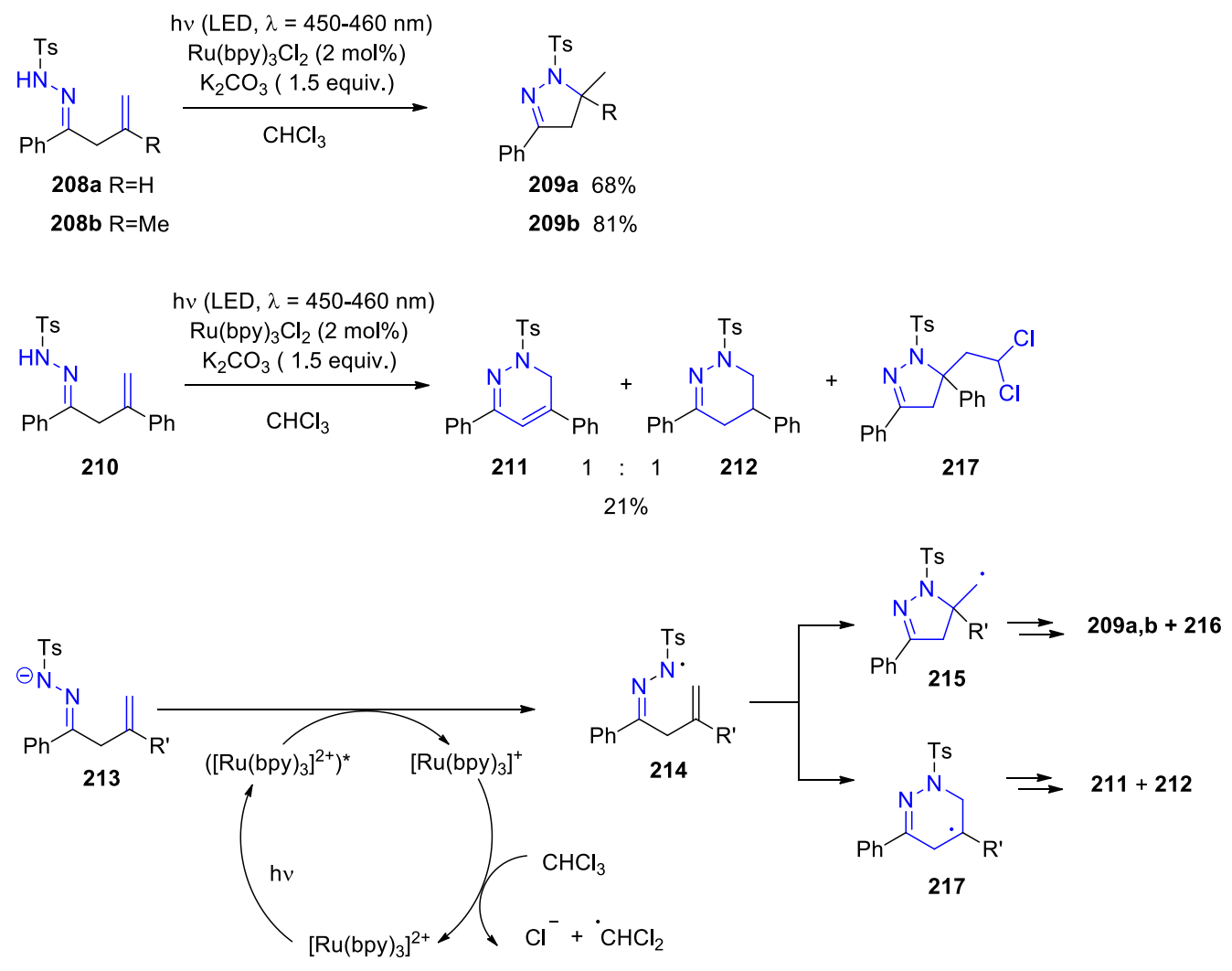

Scheme 37. Formation of dihydropyrazoles, dihydropyridazines or tetrahydropyridazines from hydrazones.

In similar reactions carried out in a basic medium, aromatic compounds $\mathbf{2 1 8}$ carrying an alkyne function and a hydrazinyl aryl sulfone group are transformed into phthalazines $\mathbf{2 1 9}$ (Scheme 38). ${ }^{83}$ In the present paper, 25 derivatives have been successfully transformed and the products have been isolated with yields up to $83 \%$. This compound family is interesting due to their pharmaceutical properties such as bactericidal or antitumor activities.

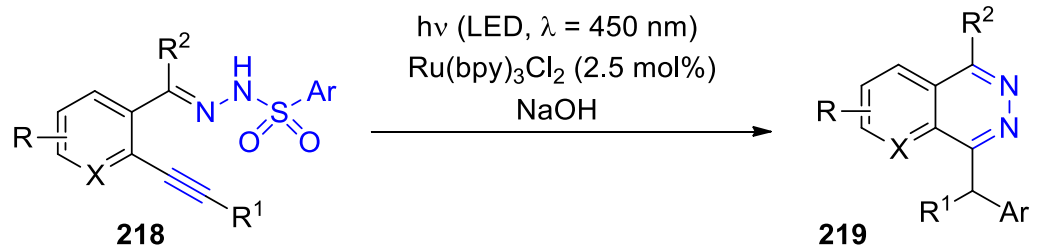

Scheme 38. Photoredox catalytic synthesis of phthalazines.

Again, a nitrogen centered radical $\mathbf{2 2 1}$ is generated by oxidation of the corresponding anion $\mathbf{2 2 0}$ using photoredox catalysis with $\mathrm{Ru}(\mathrm{bpy})_{3} \mathrm{Cl}_{2}$ (Scheme 39). In a first cyclization, the racial intermediate $\mathbf{2 2 2}$ is 
generated. In a Smiles rearrangement, $\mathrm{SO}_{2}$ is eliminated, and a second cyclization takes place which leads to the radical $\mathbf{2 2 3}$ or $\mathbf{2 2 3}$. The latter intermediate is reduced by electron transfer from the Ru(I) species generated in the photoredox catalytic cycle (224). Protonation occurs in the diarylmethyl position of 224 and not at the nitrogen atom of the phthalazine ring. Deuterium labeling experiences showed that a proton or a deuterium is transferred from the solvent. Similar reactions have been recently reported. ${ }^{84}$

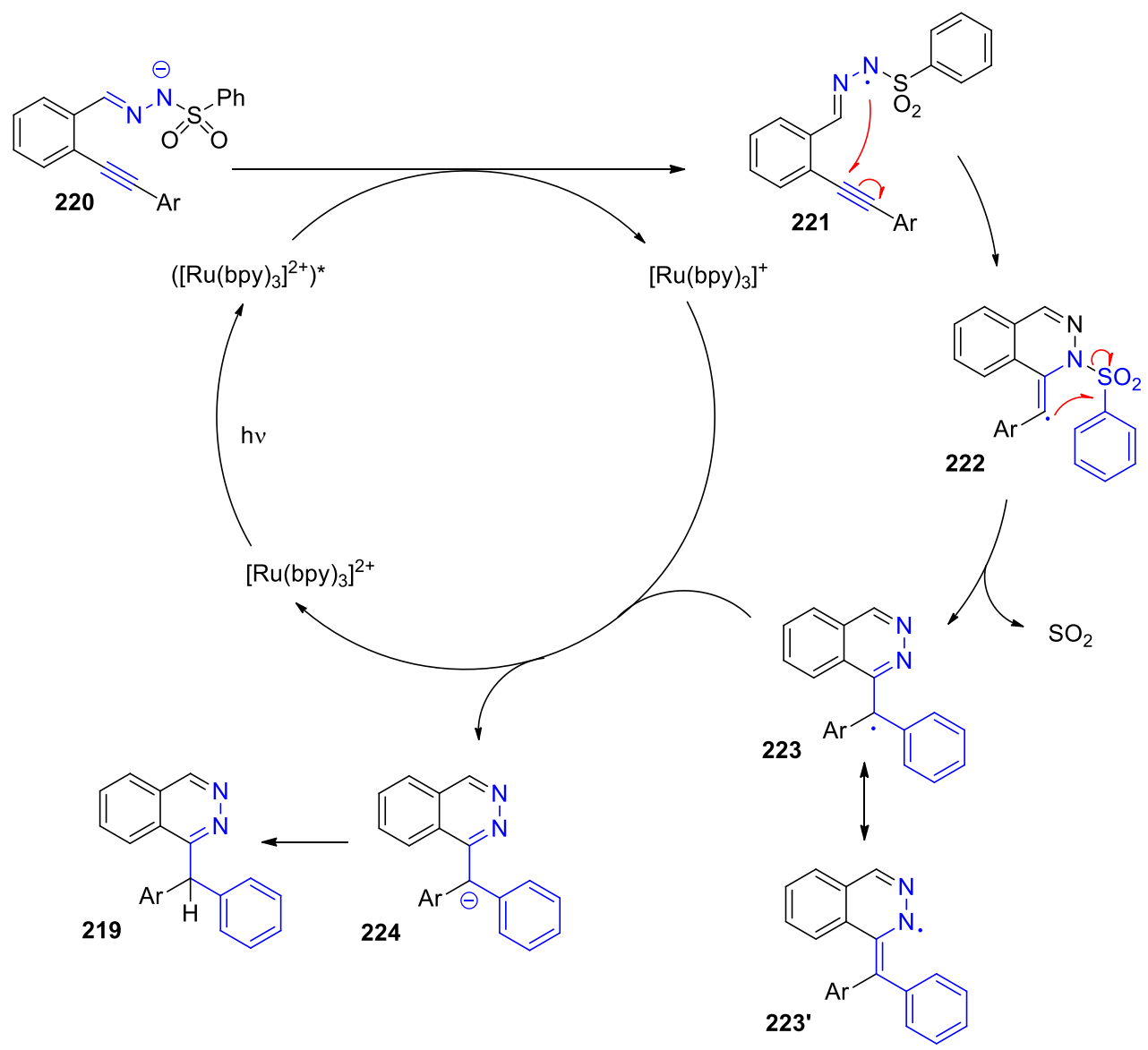

Scheme 39. Mechanism of Photoredox catalytic synthesis of phthalazines involving a Smiles rearrangement.

\section{Conclusion}

Imines and related compounds with a $\mathrm{C}=\mathrm{N}$ double bond such as oximes or hydrazones play a key role in the synthesis of numerous heterocycles. The synthesis methodology is considerably enriched by radical reactions. It is well established that photochemical reactions give access to a large variety of radical intermediates. Thus toxic reagents, typically used in many ground state radical reactions are avoided. In this way, radical chemistry becomes more and more sustainable. Since about 15 years in academic research and for some years now in chemical industry, photochemical reactions experience a renaissance in the field of organic synthesis. This impact originates mainly from numerous research activities in the domain of photoredox catalysis, especially with visible light. More recently, systematic studies on the excitation of charge transfer complexes to generate reactive intermediates have been 
carried out. These developments also push radical chemistry so that it will significantly gain importance in organic synthesis.

\section{Conflicts of interest}

There are no conflicts to declare.

\section{Acknowledgements}

Our current research in the field of imine photochemistry is supported by the Agence Nationale de Recherche (ANR, Project IMPHOCHEM).

\section{Notes and references}

1 A. F. Parsons, An introduction to free radical chemistry, Blackwell Science Ltd., Oxford, 2000. J. Fossey, D. Lefort and J. Sorba, Free radicals in organic chemistry, Wiley, Chichester, 1995.

$2 \mathrm{H}$. Fischer and L. Radom, Factors controlling the addition of carbon-centered radicals to alkenes an experimental and theoretical perspective, Angew. Chem. Int. Ed., 2001, 40, 1340-1371. B. P. Roberts, Polarity-reversal catalysis of hydrogen-atom abstraction reactions: concepts and applications in organic chemistry, Chem. Soc. Rev. 1999, 28, 25-35. D. Ravelli, M. Fagnoni, T. Fukuyama, T. Nishikawa and I. Ryu, Site-Selective C-H Funtionalization by Decatungstate Anion Photocatalysis: Syntergistic Control by Polar and Steric Effects, ACS Catal. 2018, 8, 701-713.

3 P. A. Baguley and J. C. Walton, Flight form the Tyranny of Tin: The Quest for Practical Radical Sources Free from Metal Encumbrances, Angew. Chem. int. Ed., 1998, 37, 3072-3082.

4 V. T. Perchyonok, I. Lykakis and A. Postigo, Streamlining Free Radical Green Chemistry, RSC Publishing, Cambridge, 2012.

5 H. E. Bonfield, T. Knauber, F. Lévesque, E. G. Moschetta, F. Susanne and L. J. Edwards, Photons as a 21st century reagent, Nat. Commun., 2020, 11, 804. C. Michelin and N. Hoffmann, Photocatalysis applied to organic synthesis - A green chemistry approach. Curr. Opin. Green Sustainable Chem. 2018, 10, 40-45. N. Hoffmann. Photochemical reactions of aromatic compounds and the concept of the photon as a traceless reagent, Photochem. Photobiol. Sci., 2012, 11, 1613-1641. M. Oelgemöller, C. Jung and J. Mattay, Green photochemistry: Production of fine chemicals with sunlight. Pure Appl. Chem. 2007, 79, 1939-1947. N. J. Turro and G. Schuster. Photochemical Reactions as a tool in organic synthesis, Science, 1975, 187, 303-312.

6 N. Hoffmann, Photochemical reactions as key steps in organic synthesis, Chem. Rev., 2008, 108, 1052-1103. T. Bach and J. P. Hehn, Photochemical reactions as key steps in Natural Product Synthesis. Angew. Chem. Int. Ed., 2011, 50, 1000-1052. M. D. Kärkäs, J. A. Porco and C. R. J. Stephenson, Photochemical Approaches to Complex Chemotypes: Application in Natural Product Synthesis, Chem. Rev., 2016, 116, 9683-9747.

7 B. König (Ed.), Chemical Photocatalysis, 2nd Edition, De Gruyter, Berlin, 2020. C. R. J. Stephenson, T. P. Yoon and D. W. C. MacMillan (Eds.), Visible light photocatalysis in organic chemistry, WileyVCH, Weinheim, 2018. N. A. Romero, D. A. Nicewicz, Organic Photoredox Catalysis, Chem. Rev. 2016, 116, 10075-10166. L. Capaldo and D. Ravelli, Hydrogen Atom Transfer (HAT): A Versatile Strategy for Substrate Activation in Photocatalyzed Organic Synthesis, Eur. J. Org. Chem. 2017, 2017, 2056-2071. 
8 For a selection of reviews see: A. Studer and D. Curran, Catalysis of Radical Reactions: A Radical Chemistry Perspective. Angew. Chem. Int. Ed. 2016, 55, 58-102. C. H. Schiesser, U. Wille, H. Matsubara and I. Ryu, Electrophiles: Dual Orbital Effects in Nitrogen-Philic Acyl Radical Cyclization and Related Addition Reactions. Acc. Chem. Res. 2007, 40, 303-313. A. Baralle, A. Baroudi, M. Daniel, L. Fensterbank, J.-P. Goddard, E. Lacôte, M.-H. Larraufie, G. Maestri, M. Malacria and C. Ollivier, Radical Cascade Reactions. In Encyclopedia of Radicals in Chemistry, Biology and Materials, Vol. 2, (D. Chatgilialoglu and A. Studer, Eds.), Wiley \& Sons Ltd. 2012, p. 767-816. K. Gilmore and I. V. Alabugin, Unusual Cyclizations. In Encyclopedia of Radicals in Chemistry, Biology and Materials, Vol. 2, (D. Chatgilialoglu and A. Studer, Eds.), Wiley \& Sons Ltd. 2012, p. 693-728. E. Riguet and N. Hoffmann, Synthetic Radical Photochemistry. In Encyclopedia of Radicals in Chemistry, Biology and Materials, Vol. 2, (D. Chatgilialoglu and A. Studer, Eds.), Wiley \& Sons Ltd. 2012, p.1217-1241. B. Giese, B. Kopping, T. Göbel, J. Dickhaut, G. Thoma, K. J. Kulicke and F. Trach, Radical Cyclization Reactions. Org. React. 1996, 48, 301-356. W. R. Bowman, C. F. Bridge and P. Brookes, Synthesis of heterocycles by radical cyclisation. J. Chem. Soc., Perkin Trans. 1, 2000, 1-14. W. R. Bowman, M. O. Cloonan and S. L. Krintel, Synthesis of heterocylces by radical cyclisation. J. Chem. Soc., Perkin Trans. 1, 2001, 2885-2902. S. Z. Zard, The Xanthate Route to Indolines, Indoles, and their Aza Congeners. Chem. Eur. J. 2020, 26, 12689-12705. M. P. Sibi, S. Manyem and J. Zimmerman, Enantioslective Radical Processes. Chem. Rev. 2003, 103, $3263-$ 3295. U. Jahn, J.-M. Galano and T. Durand, Beyond Prostagandins - Chemistry and Biology of Cyclic Oxygenated Metabolites Formed by Free-Radical Pathways from Polyunsaturated Fatty Acids. Angew. Chem. Int. Ed. 2008, 47, 5894-5955. P. Renaud, Radical reaction as a tool for natural product synthesis. Chimia 2001, 55, 1045-1048. J.-R. Chen, X.-Y. Yu, W. J. Xiao, Tandem Radical Cyclization of N-Arylacrylamides: An Emerging Platform for the Construction of 3,3Disubstituted Oxindoles. Synthesis 2015, 47, 604-629.

9 C. Lefebvre, L. Fortier and N. Hoffmann, Photochemical rearrangements in heterocyclic chemistry, Eur. J. Org. Chem., 2020, 2020, 1393-1404.

10 F. Dénès, Heteroatom-Centered Radicals for the Synthesis of Heterocyclic Compounds. Top. Heterocycl. Chem. 2018, 54, 151-230

11 R. W. Layer, The Chemistry of Imines. Chem. Rev. 1962, 62, 489-510. W. Qin, S. Long, M. Panunzio and S. Biondi, Schiff Bases: A Short Survey on an Evergreen Chemistry Tool. Molecules 2013, 18, 12264-12289. M. T. Nazeri, H. Farhid, R. Mohammadian and A. Shaabani, Cyclic Imines in Ugi and Ugi-Type Reaction. ACS Comb. Sci. 2020, 22, 361-400.

12 M. J. Tomaszewski, J. Warkentin and N. H. Werstiuk, Free-Radical Chemistry of Imines. Aust. J. Chem. 1995, 48, 291-321.

$13 \mathrm{X}$. Jie, Y. Shang, Z.-N. Chen, X. Zhang, W. Zhuang and W. Su. Differentiation between enamines and tautomerizable imines in the oxidation reaction with TEMPO. Nat. Commun. 2018, 9, 5002. A. Amar, H. Meghezzi, A. Boucekkine, R. Kaoua and B. Kolli. How to drive imine-enamine tautomerism of pyronic derivatives of biological interest - A theoretical and experimental study of substituent and solvent effects. C. R. Chimie 2010, 13, 553-560.

14 G. Wettermark, Photochemistry of the carbon-nitrogen double bond, In The chemistry of carbonnitrogen double bond (S. Patai, Ed.), John Wiley \& Sons, London, 1970, pp. 565-596. H. Suginome, E,Z-Isomerization and Accompanying Photoreactions of Oximes, Oxime Ethers, Nitrones, Hydrazones, Imines, Azo- and Azoxy Compounds, and Various Applications. In CRC Handbook of 
Organic Photochemistry and Photobiology, $2^{\text {nd }}$ Edition. (W. Horspool and F. Lenci, Eds.), CRC Press, Boca Raton, 2004, pp. 94/1-94/55.

15 D. Uraguchi, Y. Tsuchiya, T. Ohtani, T. Enomoto, S. Masaoka, D. Yokogawa and T. Ooi, Unveiling Latent Photoreactivity of Imines. Angew. Chem. Int. Ed. 2020, 59, 3665-3670.

16 A. Padwa, Photochemistry of the carbon-nitrogen double bond, Chem. Rev. 1977, 77, 37-68; A.

C. Pratt, The photochemistry of imines, Chem. Soc. Rev., 1977, 6, 63-81.

17 S. K. Kandappa, L. K. Valloli, S. Ahuja, J. Parthiban and J. Sivaguru, Taming the excited state reactivity of imines - from non-radiative decay to aza Paterno-Büchi reaction, Chem. Soc. Rev. 2021, DOI 10.1039/d0cs00717j

18 D. Staveness, J. L. Collins III, R. C. McAtee and C. R. J. Stephenson, Exploiting imine photochemistry for masked N-centered radical reactivity, Angew. Chem. Int. Ed. 2019, 58, 19000-19006.

19 P. J. Campos, A. Soldevilla, D. Sampedro and M. A. Rodríguez, N-cyclopropylimine-1-pyrroline rearrangement. A novel photochemical reaction, Org. Lett. 2001, 3, 4087-4089.

20 D. Sampedro, A. Soldevilla, M. A. Rodríguez, P. J. Campos and M. Olivucci, Mechanism of the Ncyclopropylimine-1-pyrroline photorearrangement, J. Am. Chem. Soc. 2005, 127, 441-448; A. Soldevilla, D. Sampedro, P. J. Campos and M. A. Rodríguez, The N-cyclopropylimine-1-pyrroline photorearrangement as a synthetic tool: scope and limitations, J. Org. Chem. 2005, 70, 69766979.

21 M. Sowden, J. L. Collins III, D. Staveness and C. R. J. Stephenson, 2020, A one pot photochemical method for the generation of functionalized aminocyclopentanes. ChemRxiv. Preprint. https://doi.org/10.26434/chemrxiv.13079159.v1

22 A. S. Harmata, M. J. Sowden and C. R. J. Stephenson, Bridged bicyclic building block upgrading: Photochemical synthesis of bicyclo[3.1.1] heptan-1-amines, 2020, ChemRxiv. Preprint. https://doi.org/10.26434/chemrxiv.13118528.v1

23 O. A. Mukhina, D. M. Kuznetsov, T. M. Cowger and A. G. Kutateladze, Amino azaxylylenes photogenerated from o-amido imines: photoassisted access to complex spiro-poly-heterocycles, Angew. Chem. Int. Ed. 2015, 54, 11516-11520.

24 O. A. Mukhina, W. C. Cronk, N. N. B. Kumar, M. C. Sekhar, A. Samanta and A. G. Kutateladze, Intramolecular cycloadditions of photogenerated azaxylylenes: An experimental and theoretical study, J. Phys. Chem. A 2014, 118, 10487-10496; O. A. Mukhina, N. N. B. Kumar, T. M. Arisco, R. A. Valiulin, G. A. Metzel and A. G. Kutateladze, Rapid photoassisted access to N,O,Spolyheterocycles with benzoazocine and hydroquinoline cores: Intramolecular cycloadditions of photogenerated azaxylylenes, Angew. Chem. Int. Ed. 2011, 50, 9423 -9428.

25 C. Lefebvre, C. Michelin, T. Martzel, V. D. Mvondo, V. Bulach, M. Abe and N. Hoffmann, Photochemically induced intramolecular radical cyclization reactions with imines, J. Org. Chem. 2018, 83, 1867-1875.

26 M. Mella, E. Fasani and A. Albini, Electron Transfer Photoinduced Cleavage of Acetals. A Mild Preparation of Alkyl Radicals, J. Org. Chem. 1992, 57, 3051-3057. M. Mella, M. Fagnoni, M. Freccero, E. Fasani and A. Albini, New synthetic methods via radical cation fragmentation, Chem. Soc. Rev. 1998, 27, 81-89. 
27 N. Hoffmann, Electron and hydrogen transfer in organic photochemical reactions, J. Phys. Org. Chem. 2015, 28, 121-136. N. Hoffmann, Photochemical Electron and Hydrogen Transfer in Organic Synthesis: The Control of Selectivity, Synthesis 2016, 48, 1782-1802.

28 R. Jahjah, A. Gassama, V. Bulach, C. Suzuki, M. Abe, N. Hoffmann, A. Martinez and J.-M. Nuzillard, Stereoselective Triplet-Sensitized Radical Reactions of Furanone Derivatives, Chem. Eur. J. 2010, 16, 3341-3354. G. Lejeune, J. Font, T. Parella, R. Alibés and M. Figueredo, Intramolecular Photoreactions of (5S)-5-Oxymethyl-2(5H)-furanones as a Tool for the Stereoselective Generation of Diverse Polycyclic Scaffolds, J. Org. Chem. 2015, 80, 9437-9445.

29 S.-Y. Hsieh and J. W. Bode, Silicon amine reagents for the photocatalytic synthesis of piperazines from aldehydes and ketones, Org. Lett. 2016, 18, 2098-2101.

30 S.-Y. Hsieh and J. W. Bode, Lewis acid induced toggle from $\operatorname{Ir}(\mathrm{II})$ to $\operatorname{Ir}(\mathrm{IV})$ pathways in photocatalytic reactions: synthesis of thiomorpholines and thiazepanes from aldehydes and SLAP reagents, ACS Cent. Sci. 2017, 3, 66-72; M. K. JackI, L. Legnani, B. Morandi and J. W. Bode, Continuous flow synthesis of morpholines and oxazepanes with silicon amine protocol (SLAP) reagents and Lewis acid facilitated photoredox catalysis, Org. Lett. 2017, 19, 4696-4699.

31 N. B. Bissonnette, J. M. Ellis, L. G. Hamann and F. Romanov-Michailidis, Expedient access to saturated nitrogen heterocycles by photoredox cyclization of imino-tethered dihydropyridines, Chem. Sci., 2019, 10, 9591-9596.

32 D. C. Miller, K. T. Tarantino and R. R. Knowles, Proton-Coupled Electron Transfer in Organic Synthesis: Fundamentals, Applications, and Opportunities. Top. Curr. Chem. 2016, 374, 30. N. Hoffmann, Proton-Coupled Electron Transfer in Photoredox Catalytic Reactions. Eur. J. Org. Chem. 2017, 2017, 1982-1992. L. Q. Nguyen and R. R. Knowles, Catalytic C-N Bond-Forming Reactions Enabled by Proton-Coupled Electron Transfer Activation of Amide N-H Bondes. ACS Catal. 2016, 6, 2894-2903.

33 L. R. E. Pantaine, J. A. Milligan, J. K. Matsui, C. B. Kelly, and G. A. Molander, Photoredox radical/polar crossover enables construction of saturated nitrogen heterocycles, Org. Lett. 2019, 21, 2317-2321.

34 V. Corcé, L.-M. Chamoreau, E. Derat, J.-P. Goddard, C. Ollivier and L. Fensterbank, Silicates as Latent Alkyl Radical Precursors: Visible-Light Photocatalytic Oxidation of Hypervalent BisCatecholato Silicon Compounds, Angew. Chem. Int. Ed. 2015, 54, 11414-11418.

35 L. Wang, Z.-G. Ma, X.-J. Wei, Q.-Y. Meng, D.-T. Yang, S.-F. Du, Z.-F. Chen, L.-Z. Wu and Q. Liu, Synthesis of 2-substituted pyrimidines and benzoxazoles via a visible-light-driven organocatalytic aerobic oxidation: enhancement of the reaction rate and selectivity by a base, Green Chem., 2014, 16, 3752-3757.

36 Y.-X. Chen, L.-F. Qian, W. Zhang and B. Han, Efficient Aerobic Oxidative Synthesis of 2-Substituted Benzoxazoles, Benzothiazoles, and Benzimidazoles Catalyzed by 4-Methoxy-TEMPO, Angew. Chem. Int. Ed. 2008, 47, 9330 -9333.

37 Selected examples: A. Padwa, Azirine photochemistry, Acc. Chem. Res. 1976, 9, 371-378; E. Albrecht, J. Averdung, E. W. Bischof, A. Heidbreder, T. Kirschberg, F. Müller and J. Mattay, Photoinduced electron transfer (PET) in organic synthesis. [3 + 2]-type cycloaddition, cyclization and C-C bond cleavage reactions, J. Photochem. Photobiol., A 1994, 82, 219-232; B. Jackson, M. Märky, H.-J. Hansen and H. Schmid, Photochemische Cycloadditionen von 3-Phenyl-2H-azirinen mit aktivierten Doppelbindungen, Helv. Chim. Acta 1972, 55, 919- 921; F. Müller, J. Mattay and 
Steen Steenken, Radical ions and photochemical charge-transfer phenomena. 39. Cycloadditions. 42. Radical cation [3 + 2] cycloadditions of $2 \mathrm{H}$-azirines. Mechanistic studies concerning the intermediate radical cation, J. Org. Chem. 1993, 58, 4462-4464; H. Inui and S. Murata, Photochemistry of 2-(1-Naphthyl)-2H-azirines in Matrixes and in Solutions: WavelengthDependent C-C and C-N Bond Cleavage of the Azirine Ring, J. Am. Chem. Soc. 2005, 127, 26282636; D. W. Gamage, Q. Li, R. A. A. U. Ranaweera, S. K. Sarkar, G. K. Weragoda, P. L. Carr and A. D. Gudmundsdottir, Vinylnitrene Formation from 3,5-Diphenyl-isoxazole and 3-Benzoyl-2phenylazirine, J. Org. Chem. 2013, 78, 11349-11356.

38 F. Müller and J. Mattay, A new synthesis for imidazolo- and pyrrolophanes by [3+2] cycloaddition with azaallenyl radical cations, Angew. Chem. Int. Ed. Engl. 1991, 30, 1336-1337.

39 T.-T. Zeng, J. Xuan, W. Ding, K. Wang, L.-Q. Lu and W.- J. Xiao, [3 + 2] cycloaddition/oxidative aromatization sequence via photoredox catalysis: one-pot synthesis of oxazoles from $2 \mathrm{H}$-azirines and aldehydes, Org. Lett., 2015, 17, 4070-4073.

40 J. Xuan, X.-D. Xia, T.-T. Zeng, Z.-J. Feng, J.-R. Chen, L.-Q. Lu and W.-J. Xiao, Visible-light-induced formal [3+2] cycloaddition for pyrrole synthesis under metal-free conditions, Angew. Chem. Int. Ed. 2014, 53, $5653-5656$.

41 J. A. Leitch, A. L. Fuentes de Arriba, J. Tan, O. Hoff, C. M. Martinez and D. J. Dixon, Photocatalytic reverse polarity Povarov reaction, Chem. Sci., 2018, 9, 6653-6658.

42 W-F. Tian, D-P. Wang, S-F. Wang, K-H. He, X-P. Cao and Y. Li, Visible-light photoredox-catalyzed iminyl radical formation by $\mathrm{N}-\mathrm{H}$ cleavage with hydrogen release and its application in synthesis of isoquinolines, Org. Lett. 2018, 20, 1421-1425.

$43 \mathrm{X}$. Hu, G. Zhang, F. Bu, and A. Lei, Selective Oxidative [4+2] Imine/Alkene Annulation with H2 Liberation Induced by Photo-Oxidation, Angew. Chem. Int. Ed. 2018, 57, 1286 -1290.

44 M. Minozzi, D. Nanni and P. Spagnolo, Imidoyl radicals in organic synthesis, Curr. Org. Chem., 2007, 11, 1366-1384; J. Lei, J. Huang and Q. Zhu, Recent progress in imidoyl radical-involved reactions, Org. Biomol. Chem., 2016, 14, 2593-2602.

45 X. Dong, Y. Xu, J. J. Liu, Y. Hu, T. Xiao and L. Zhou, Visible-light-induced radical cyclization of trifluoroacetimidoyl chlorides with alkynes: catalytic synthesis of 2-trifluoromethyl quinolines, Chem. Eur. J. 2013, 19, 16928-16933.

46 X. Dong, Y. Hu, T. Xiao and L. Zhou, Synthesis of 2-trifluoromethyl indoles via visiblelight induced intramolecular radical cyclization, RSC Adv., 2015, 5, 39625-39629.

47 W. Fu, M. Zhu, F. Xu, Y. Fu, C. Xu and D. Zou, An approach to 6-trifluoromethyl-phenanthridines through visible-light-mediated intramolecular radical cyclization of trifluoroacetimidoyl chlorides, RSC Adv., 2014, 4, 17226-17229.

48 J. C. Walton, Functionalised Oximes: Emergent Precursors for Carbon-, Nitrogen- and OxygenCentered Radicals. Molecules 2016, 21, 63/1-63/23. J. C. Walton, Synthetic Strategies for 5- and 6-Membered Ring Azaheterocycles Facilitated by Iminyl Radicals. Molecules 2016, 21, 660/1660/25. F. Xiao, Y. Guo, Y.-F. Zeng, Recent Developments in Radical Cross-Coupling of RedoxActive Cycloketone Oximes, Adv. Synth. Catal. 2021, 363, 120-143. W. Yin, X. Wang, Recent Advances in Iminyl Radical-mediated Catalytic Cyclizations and Ring-Opening Reactions. New J. Chem. 2019, 43, 3254-3264. P. Wang, Q. Zhao, W. Xiao, J. Chen, Recent advances in visible-light photoredox-catalyzed nitrogen radical cyclization. Green Synth. Catal. 2020, 1, 42-51. 
49 H. Sakuragi, S. Ishikawa, T. Nishimura, M. Yoshida, N. Inamoto and K. Tokumaru, Homolytic Aromatic Substitution by Iminyl Radicals. Photolysis of Aromatic Ketone O-Acyloximes in Aromatic Solvents. Bull. Chem. Soc. Jpn. 1976, 49, 1949-1954.

50 M. Fréneau, P. de Sainte Claire, N. Hoffmann, J.-P. Vors, J. Geist, M. Euvrard and C. Richard, Phototransformation of tetrazoline oxime ethers: photoisomerization vs. photodegradation. RSC Adv. 2016, 6, 5512-5522. M. Fréneau, N. Hoffmann, J.-P. Vors, P. Genix, C. Richard and P. de Sainte Claire, Phototransformation of tetrazoline oxime ethers - part2: theoretical investigation. RSC Adv. 2016, 6, 63965-63972.

51 J. L. Hofstra, B. R. Grassbaugh, Q. M. Tran, N. R. Armada and H. J. P. de Lijser, Catalytic Oxidative Cyclization of 2-Arylbenzaldehyde Oxime Ethers under Photoinduced Electron Transfer Conditions. J. Org. Chem. 2015, 80, 256-265.

52 R. T. McBurney and J. C. Walton, Interplay of ortho- with spiro-cyclisation during iminyl radical closures onto arenes and heteroarenes. Beilstein J. Org. Chem. 2013, 9, 1083-1092. R. T. McBurney, A. M. Z. Slawin, L. A. Smart, Y. Yu and J. C. Walton, UV promoted phenanthridine syntheses from oxime carbonate derived iminyl radicals. Chem. Commun. 2011, 47, 7974-7976.

53 R. Alonso, A. Caballero, P. J. Campos, M. A. Rodríguez, Photochemistry of acyloximes: synthesis of heterocycles and natural products. Tetrahedron 2010, 66, 8828-8831.

54 R. Alonso, P. J. Campos, B. García and M. Rodríguez, New Light-Induced Iminyl Radical Cyclization Reactions of Acyloximes to Isoquinolines. Org. Lett. 2006, 8, 3521-3523.

55 Y. Han, Y. Jin, M. Jiang, H. Yang and H. Fu, Photocatalist-Free Visible-Light Photoredox Dearomatization of Phenol Derivatives Containing Ketoximes: An Easy Access to Spiropyrrolines. Org. Lett. 2019, 21, 1799-1803.

56 J. Davis, S. G. Booth, S. Essafi, R. A. W. Dryfe and D. Leonori, Visible-Light-Mediated Synthesis of Amidyl Radicals: Transition-Metal-Free Hydroamination and N-Arylation Reactions. Angew. Chem. Int. Ed. 2015, 54, 14017-14021.

57 For recent examples of photochemical reactions induced by charge transfer or donor acceptor complexes light absorption see: G. E. M. Crisenza, D. Mazzarella and P. Melchiorre, Synthetic Methods Driven by the Photoactivity of Electron Donor-Acceptor Complexes. J. Am. Chem. Soc. 2020, 142, 5461-5476.

58 J. Li, P. Zhang, M. Jinang, H. Yang, Y. Zhao and H. Fu, Visible Light as a Sole Requirement for the Intramolecular C(sp $\left.{ }^{3}\right)$-H Imination. Org. Lett. 2017, 19, 1994-1997.

59 R. Mao, Z. Yuan, Y. Li and J. Wu, N-Radical-Initiated Cyclization through Insertion of Sulfur Dioxide under Photoinduced Catalyst-Free Conditions. Chem. Eur. J. 2017, 23, 8176-8179. 60 X. D. An and S. Yu, Visible-Light-Promoted and One-Pot Synthesis of Phenanthridines and Quinolines form Aldehydes and O-Acyl Hydroxylamine. Org. Lett. 2015, 17, 2692-2695.

$61 \mathrm{H}$. Jiang, X. An, K. Tong, T. Zheng, Y. Zhang and S. Yu, Visible-Light-Promoted Iminyl-Radical Formation form Acyl Oximes: A Unified Approach to Pyridines, Quinolines, and Phenanthridines. Angew. Chem. Int. Ed. 2015, 54, 4055-4059.

62 T. Mikami and K. Narasaka, Photochemcial Transformation of $\gamma, \delta$-Unsaturated Ketone O-(pCyanophenyl)oximes to 3,4-Dihydro-2H-Pyrrole Derivatives. Chem. Lett. 2000, 29, 338-339. T. Mikami and K. Narasaka, Photochemcial transformation of $\gamma, \delta$-unsaturated ketone $O-(p-$ cyanophenyl)oximes to 3,4-dihydro-2H-pyrrole derivatives. C. R. Acad. Sci., Ser. Ilc: Chim. 2001, 4, 
477-486. M. Kitamura, Y. Mori and K. Narasaka, Photochemical radical cyclization of $\gamma, \delta$ unsaturated ketone oximes to 3,4-dihydro-2H-pyrroles. Tetrahedron Lett. 2005, 46, 2373-2376.

63 K. Usami, E. Yamaguchi, N. Tada and A. Itoh, Visible-Light-Mediated iminyl Radical Generation from Benzyl Oxime Ether: Synthesis of Pyrroline via Hydroimination Cyclization. Org. Lett. 2018, 20, 5714-5717.

64 R. Alonso, P. J. Campos, M. A. Rodríguez and D. Sampedro, Photocyclization of Iminyl Radicals: Theoretical Study and Photochemcial Aspects. J. Org. Chem. 2008, 73, 2234-2239. T. T. McBurney and J. C. Walton, Dissociation or Cyclization: Options for a Triad of Radicals Released form Oxime Carbamates. J. Am. Chem. Soc. 2013, 135, 7349-7354.

$65 \mathrm{H}$. Jiang and A. Studer, Iminyl-Radicals by oxidation of $\alpha$-Imino-oxy Acids: Photoredox-Neutral Alkene Carboimination for the Syntheisis of Pyrrolines. Angew. Chem. Int. Ed. 2017, 56, 1227312276.

66 F. Portela-Cubillo, J. Lymer, E. M. Scanalan, J. S. Scott and J. C. Walton, Dioxime oxalates; new iminyl radical precursors for syntheses of $\mathrm{N}$-heterocycles. Tetrahedron 2008, 64, 11908-11916.

67 J. Boivin, E. Fouquet, A.-M. Schiano and S. Z. Zard, Iminyl Radicals: Part III. Further Synthetically Useful Sources of Iminyl Radicals. Tetrahedron 1994, 50, 1769-1776.

68 S.-H. Cai, J.-H. Xie, S. Song, L. Ye, C. Feng and T.-P. Loh, Visible-Light-Promoted Carboimination of Unactivated Alkenes for the Synthesis of Densely Functionalized Pyroline Derivatives. ACS Catal. 2016, 6, 5571-4474.

69 X. Shen, C. Huang, X.-A. Yuan and S.Yy, Diastereoselective and Stereodivergent Synthesis of 2Cinnamylpyrrolidines Enabled by Photoredox-Catalyzed Iminoalkenylation of alkenes. Angew. Chem. Int. Ed. 2021, 60, 9672-9679.

70 J. Davies, N. S. Sheik and D. Leonori, Photoredox Imino Functionalization of Olefines. Angew. Chem. Int. Ed. 2017, 56, 13361-13365.

71 N. Hoffmann, Combining Photoredox and Metal Catalysis. ChemCatChem. 2015, 7, 393-394.

72 J.-L. Tu, J.-L. Liu, W. Tang, M. Su and F. Liu, Radical Aza-Cyclization of $\alpha$-Imino-oxy Acids for Synthesis of Alkene-Containing N-Heterocycles via Dual Cobaloxime and Photoredox Catalysis. Org. Lett. 2020, 22, 1222-1226.

73 C. L. Cavanaugh and D. A. Nicewicz, Synthesis of $\alpha$-Benzyloxyamino- $\gamma$-butyrolactones via Polar Radical Crossover Cycloaddition Reaction. Org. Lett. 2015, 17, 6082-6085.

74 F. Dénès, M. Pichowicz, G. Povie and P. Renaud, Thiyl Radicals in Organic Synthesis, Chem. Rev. 2014, 114, 2587-2693.

75 D. Li, H. Ma and W. Yu, Visible Light-Induced Radical Cyclization of Ethyl 2-(N-Arylcarbamoyl)-2Chloroiminioacetates: Synthesis of Quinoxalin-2(1H)-ones. Adv. Synth. Catal. 2015, 357, 36963702.

76 G. K. Friestad, Addition of carbon-centered radicals to imines and related compounds, Tetrahedron 2001, 57, 5461-5496.

77 L. J. Rono, H. G. Yayla, D. Y. Wang, M. F. Armstrong and R. R. Knowles, Enantioselective Photoredox Catalysis Enabled by Proton-Coupled Electron Transfer: Development of an Asymmetric Aza-Pinacol Cyclization. J. Am. Chem. Soc. 2013, 135, 17735-17738.

78 N. Zhou, J. Liu, Z. Yan, Z. Wu, H. Zhang W. Li and C. Zhu, Synthesis of cyclohexylidenehydrazinefuced polycyclics via photocatalytic radical cascade reaction of 2-ethynylaldehyd hydrazones. Chem. Commun. 2017, 53, 2036-2039. 
79 X.-Y. Yu, Q.-Q. Zhao, J. Chen, W.-J. Xiao and J.-R. Chen, When Light Meets Nitrogen-Centered Radicals: From Reagents to Catalysts. Acc. Chem. Res. 2020, 53, 1066-1083. J.-R. Chen, X.-Q. Hu, L.-Q. Lu and W.-J. Xiao, Exploration of Visible-Light Photocatalysis in Heterocycle Synthesis and Functionalization. Acc. Chem. Res. 2016, 49, 1911-1923.

80 X.-Q. Hu, X. Qi, J.-R. Chen, Q.-Q. Zhao, Q. Wei, Y. Lan and W.-J. Xiao, Catalytic N-radical cascade reaction of hydrazones by oxidative deprotonation electron transfer and TEMPO mediation. Nat. Commun. 2016, 7, 11188. For a previous study on such reactions see: X.-Q. Hu, J-R. Chen, Q. Wei, F.-L. Liu, Q.-H. Deng, A. M. Beauchemin and W.-J. Xiao, Photocatalytic Generation of N-Centered Hydrazonyl Radicals: A Strategy for Hydroamination of $\beta, \gamma$-Unsaturated Hydroazones. Angew. Chem. Int. Ed. 2014, 53, 12163-12167.

81 X.-Q. Hu, J. Chen, J.-R. Chen, D.-M. Yan and W.-J. Xiao, Oranophotocatalytic Generation of N- and O-Centred Radicals Enables Aerobic Oxyamination and Dioxygenation of Alkenes. Chem. Eur. J. 2016, 22, 14141-14146.

82 Q.-Q. Zhao, J. Chen, D.-M. Yan, J.-R. Chen and W.-J. Xiao, Photocatalytic Hydrazonyl RadicalMediated Radical Cyclization/Allylation Canscade: Synthesis of Dhydropyrazoles and Tetrahydropuridazines. Org. Lett. 2017, 19, 3620-3623.

83 E. Brachet, L. Marzo, M. Selkti, B. König and P. Belmont, Visible light amination/Smiles cascade: access to phthalazine derivatives. Chem. Sci. 2016, 7, 5002-5006.

84 M. Huynh, M. De Abreu, P. Belmont and E. Brachet, Spotlight on Photoinduced Aryl Migration Reactions. Chem. J. J. 2021, 27, 3581-3607. M. De Abreu, P. Belmont and E. Brachet, LightEnabled Radical 1,4-Aryl Migration Via a Phospho-Smiles Rearrangement. J. Org. Chem. 2021, 86, 3758. M. De Abreu, M. Selki, P. Belmont and E. Brachet, Phosphoramidates as Transient Precursors of Nitrogen-Centered Radical Under Visible-Light Irradiation: Application to the Synthesis of Phthalazine Derivatives. Adv. Synth. Catal. 2020, 362, 2216-2222.

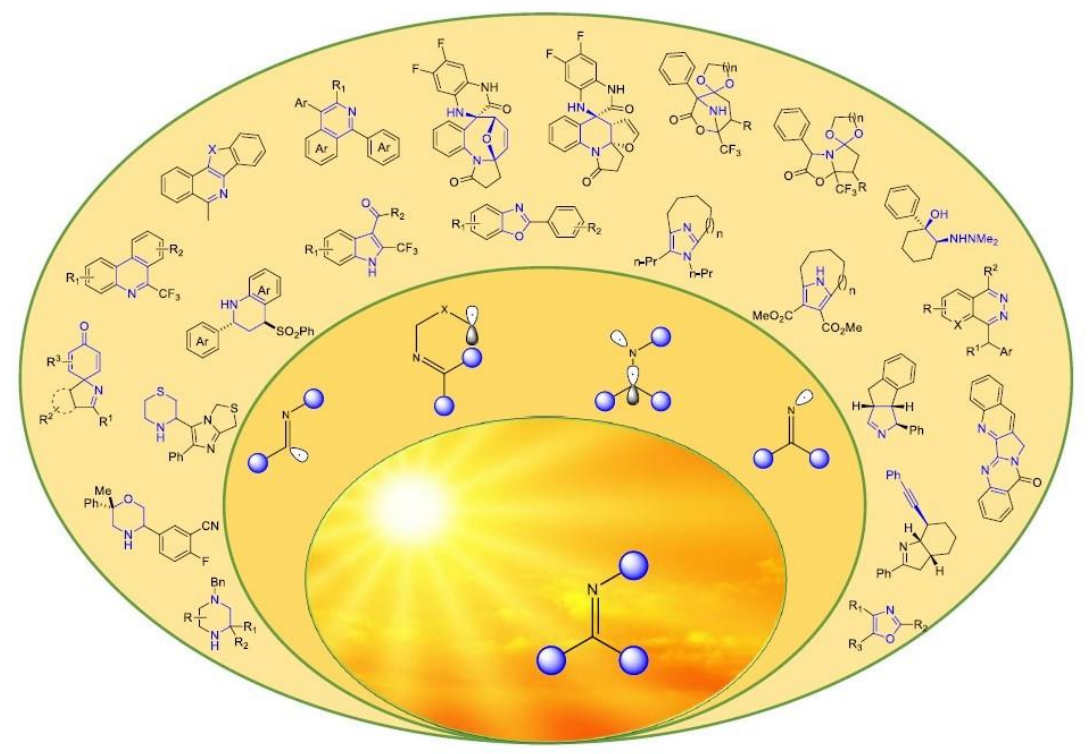

Photochemical reactions of imines, hydrazones, oximes and related Compounds provides an efficient access to radical intermediates in the synthesis of heterocycles. 